\title{
Heterogeneity of response to immune checkpoint blockade in hypermutated experimental gliomas
}

Katrin Aslan 1,2,3,14, Verena Turco 1,2, Jens Blobner ${ }^{1,2}$, Jana K. Sonner (10 1,2,15, Anna Rita Liuzzi 4,16, Nicolás Gonzalo Núñez (1) 4,16, Donatella De Feo4,16, Philipp Kickingereder ${ }^{5}$, Manuel Fischer ${ }^{5}$, Ed Green ${ }^{1,2}$, Ahmed Sadik ${ }^{6}$, Mirco Friedrich¹,2, Khwab Sanghvi ${ }^{1,2,3}$, Michael Kilian 1,2,3, Frederik Cichon ${ }^{1,2,3}$, Lara Wolf ${ }^{1,2}$, Kristine Jähne 1,2, Anna von Landenberg 1,2, Lukas Bunse (1) 1,2, Felix Sahm (1) 7,8, Daniel Schrimpf 7,8, Jochen Meyer ${ }^{7,8}$, Allen Alexander ${ }^{1,3,5}$, Gianluca Brugnara (1) ${ }^{5}$, Ralph Röth ${ }^{9}$, Kira Pfleiderer ${ }^{1,5}$, Beate Niesler (10 ${ }^{9}$, Andreas von Deimling ${ }^{7,8}$, Christiane Opitz (10 ${ }^{6}$, Michael O. Breckwoldt (1) 1,5, Sabine Heiland ${ }^{5}$, Martin Bendszus ${ }^{5}$, Wolfgang Wick (10) 10,11,12, Burkhard Becher (i) ${ }^{4} \&$ Michael Platten (1) $1,2,13 \otimes$

Intrinsic malignant brain tumors, such as glioblastomas are frequently resistant to immune checkpoint blockade (ICB) with few hypermutated glioblastomas showing response. Modeling patient-individual resistance is challenging due to the lack of predictive biomarkers and limited accessibility of tissue for serial biopsies. Here, we investigate resistance mechanisms to anti-PD-1 and anti-CTLA-4 therapy in syngeneic hypermutated experimental gliomas and show a clear dichotomy and acquired immune heterogeneity in ICB-responder and non-responder tumors. We made use of this dichotomy to establish a radiomic signature predicting tumor regression after pseudoprogression induced by ICB therapy based on serial magnetic resonance imaging. We provide evidence that macrophage-driven ICB resistance is established by CD4 $\mathrm{T}$ cell suppression and $\mathrm{T}_{\text {reg }}$ expansion in the tumor microenvironment via the PD-L1/PD-1/CD80 axis. These findings uncover an unexpected heterogeneity of response to ICB in strictly syngeneic tumors and provide a rationale for targeting PD-L1expressing tumor-associated macrophages to overcome resistance to ICB.

\footnotetext{
${ }^{1}$ DKTK Clinical Cooperation Unit Neuroimmunology and Brain Tumor Immunology, German Cancer Research Center (DKFZ), Heidelberg, Germany. 2 Department of Neurology, Medical Faculty Mannheim, MCTN, Heidelberg University, Heidelberg, Germany. ${ }^{3}$ Faculty of Biosciences, Heidelberg University, Heidelberg, Germany. ${ }^{4}$ Institute of Experimental Immunology, University of Zurich, Zurich, Switzerland. ${ }^{5}$ Department of Neuroradiology, Heidelberg University Medical Center, Heidelberg, Germany. ${ }^{6}$ Brain Cancer Metabolism Group, German Cancer Research Center (DKFZ), Heidelberg, Germany. ${ }^{7}$ DKTK Clinical Cooperation Unit Neuropathology, German Cancer Research Center (DKFZ), Heidelberg, Germany. ${ }^{8}$ Department of Neuropathology, Heidelberg University Medical Center, Heidelberg, Germany. ${ }^{9}$ nCounter Core Facility, Institute of Human Genetics, University of Heidelberg, Heidelberg, Germany.

${ }^{10}$ Department of Neurology, Heidelberg University Medical Center, Heidelberg, Germany. ${ }^{11}$ National Center for Tumor Diseases Heidelberg, DKTK, Heidelberg, Germany. ${ }^{12}$ DKTK CCU Neurooncology, DKFZ, Heidelberg, Germany. ${ }^{13}$ Helmholtz Institute for Tranlational Oncology (HI-TRON),

Mainz, Germany. ${ }^{14}$ Present address: Immatics Biotechnologies GmbH, Tübingen, Germany. ${ }^{15}$ Present address: Institute of Neuroimmunology and Multiple Sclerosis, Center for Molecular Neurobiology Hamburg, University Medical Center Hamburg-Eppendorf, Hamburg, Germany. ${ }^{16}$ These authors contributed equally: Anna Rita Liuzzi, Nicolás Gonzalo Núñez, Donatella De Feo. ${ }^{凶}$ email: m.platten@dkfz-heidelberg.de
} 
B lockade of immune-regulatory receptors, such as programmed cell death protein-1 (PD-1) and cytotoxic T-lymphocyte-associated antigen-4 (CTLA-4) mitigates T cell suppression, and restores $\mathrm{T}$ cell activation and proliferation, thereby reinvigorating antitumor immunity ${ }^{1}$. Immune checkpoint blockade (ICB) targeting PD-1 and CTLA-4 is now implemented into the standard therapies of an increasing number of tumor entities, resulting in durable responses and increased survival in a substantial number of patients ${ }^{2,3}$. While efficacy in metastatic disease to the brain indicates that the central nervous system (CNS) is not a general barrier for ICB-mediated stimulation of antitumor immunity 4,5 , evidence from randomized clinical trials suggest that primary malignant brain tumors, such as glioblastoma are largely resistant with few hypermutated glioblastoma, representing an exception ${ }^{6,7}$. Hypermutation in glioblastomas is not strictly associated with an increased intratumoral $\mathrm{T}$ cell response $\mathrm{e}^{8-10}$, indicating that hypermutation per se is not sufficient for an effective antitumor immunity induced by ICB. Contrariwise, durable responses may occur in patients with glioblastoma (GBM) without hypermutation ${ }^{11}$. Due to the overall low response rate with very few patients responding, both the establishment of predictive biomarkers and the identification of resistance mechanisms is challenging. Syngeneic orthotopic glioblastoma models have been considered insufficient models to assess interindividual heterogeneity of immune responses. To evaluate mechanisms of response and resistance to ICB, we made use of a syngeneic experimental hypermutated orthotopic glioma model exceeding 100 nonsynonymous mutations per tumor exome $\mathrm{e}^{12-14}$ to ensure sufficient immune recognition of neo-epitopes.

Here, we made use of the dichotomy of response and nonresponse to ICB in a hypermutated glioma model to develop a predictive radiomic imaging signature and to uncover cellular and molecular mechanisms of response and non-response in the glioma immune microenvironment, providing a rationale for targeting programmed death-ligand 1 (PD-L1)-expressing tumorassociated macrophages to overcome resistance to ICB.

\section{Results}

Preclinical MRI-based response evaluation for GBM immunotherapy. Combination ICB therapy targeting PD-1 and CTLA4 suppressed tumor growth of established syngeneic orthotopic mouse gliomas (Fig. 1a-d). Despite strict syngeneity of the model, we observed a dichotomy in tumor growth upon ICB therapy in ICB responder (R) and non-responder (NR) mice as monitored by serial magnetic resonance imaging (MRI; Fig. 1b, d). To evaluate the dynamics of response and resistance in individual mice, we defined preclinical MRI response criteria based on the established clinical RANO (response assessment in neurooncology) critera ${ }^{15}$. ICB response in the preclinical model was determined by the comparison of $\mathrm{d} 13$ baseline lesion volumes (MRI1) with d26 post therapy lesion volumes (MRI3) using T2weighted MRI. Assessment of lesion volumes $(V)$ and their relative increase between $\mathrm{d} 13$ and $\mathrm{d} 26$, as well as $\mathrm{d} 19$ (MRI2, during ICB therapy) and $\mathrm{d} 26$ strongly correlated with the assessment of the lesion bidimensional diameter product (area) used in RANO criteria (Supplementary Fig. 1a, b). We next aimed at translating planumetric RANO criteria to tridimensional (volumetric) response criteria by correcting for area-volume divergence (Supplementary Fig. 1c). For tridimensional response criteria, complete response (CR) was defined as relative change in lesion volume MRI3-MRI1 ( $\left.\% V_{\text {MRI3-MRI1 }}\right)$ of $-100 \%$, partial response $(\mathrm{PR})$ as $\% V_{\text {MRI3-MRI1 }} \leq-65.0 \%$ and/or $\% V_{\text {MRI3-MRI2 }} \leq$ $-65.0 \%$, stable disease (SD) as $\% V_{\text {MRI3-MRI1 }}>-65.5 \%$ and $<+$ $40 \%$, and progressive disease (PD) as $\% V_{\text {MRI3-MRI1 }} \geq+40 \%$.
Lesions with an unconfirmed progression, defined by a $\%$ $V_{\text {MRI3-MRI1 }} \geq+40 \%$ that showed a regression of at least $-30 \%$ between MRI2 and MRI3 (\% $V_{\text {MRI3-MRI2 }}$ ) were classified as SD (Fig. 1e, Supplementary Fig. 1c, right). Taking the rapid tumor progression of Gl261 tumors into account, mice with CR, PR, and SD were grouped as ICB R and mice with PD were defined as ICB NR. Response evaluation of a dataset of 212 ICB-treated (ICB) and 73 control-treated $(C)$ mice revealed a response rate of $47.64 \%$ (ICB) compared to $5.48 \%$ (C; Fig. 1f, $p>0.001$ ). Monotherapy with PD-1 blockade showed a reduced response rate $(33.33 \%$, Supplementary Fig. $1 \mathrm{~d}-\mathrm{g})$ compared to anti-PD-1 and anti-CTLA-1 combination therapy as previously described ${ }^{16}$. ICB response evaluation based on MRI data translated into a significantly enhanced survival in ICB $\mathrm{R}$ mice (Fig. 1g). Mutanome analysis of ICB R and ICB NR tumors revealed no significant difference in the number and clonality of mutations with a sufficient number of putative neo-antigens to induce tumor immunity (Fig. $1 \mathrm{~h}-\mathrm{j}$ ). The mutanome of ICB R and ICB NR tumors was heterogeneous with $23.35 \%$ of all identified mutations enriched in ICB R tumors and $19.82 \%$ of all identified mutations enriched in ICB NR tumors (Fig. 1j). Although initial tumor size weakly correlated with therapy response (Supplementary Fig. 2a), response was not restricted to small pretreatment tumor volumes and was independent of preexisting, environmental, and genetic factors, including housing or gender (Supplementary Fig. 2b, c). Notably, heterogeneity of response to ICB therapy was not restricted to experimental gliomas but also occurred in experimental syngeneic B16 melanomas (Supplementary Fig. 3a).

Radiomic evaluation of ICB response and pseudoprogression. In serial MRI, we observed evidence of pseudoprogression, where ICB therapy induced an initial increase of the measurable MR lesion between MRI1 and MRI2 followed by a rapid regression between MRI2 and MRI3 in 77.23\% of ICB R mice (growth pattern 2; G2), while only $19.80 \%$ of ICB R mice showed immediate lesion regression between MRI1 and MRI2 or response between MRI2 and MRI3 without pseudoprogression (G1; Fig. 1c; Fig. 2a). Delayed response of ICB-treated mice with pseudoprogression (G2 growth pattern) resulted in significant bigger tumor volumes on MRI3 compared to directly responding mice (G1 growth pattern; Fig. 2b). However, no significant difference in response between MRI2 and MRT3 was present between G1 and G2 ICB R (Fig. 2c), suggesting that direct response is not a prerequisite for optimal ICB response and methods to monitor pseudoprogression in ICB R are relevant to distinguish pseudoprogressing ICB R from ICB NR.

To non-invasively predict treatment response ( $\mathrm{R}$ vs. NR) and pseudoprogression in ICB-treated mice, we implemented an MRI-based radiomic approach. We calculated a set of 423 radiomic features from the T2-hyperintense tumor volume for each time point and incorporated features from MRI1 and the change in radiomic features between the MRI1 to MRI2 for radiomic signature discovery. By constructing a gradient boost classifier, we identified a radiomic signature that allowed to predict treatment response with an accuracy of $82.7 \%(95 \%$ confidence interval, 79.8-85.4\%; sensitivity: 69.8\%; and specificity: $89.9 \%)$. Predictive accuracy was significantly higher as compared to the null model (no information rate of $64.2 \%)(P<$ 0.001 ; Fig. 2d, e, Supplementary Fig. 3b). The top radiomic feature for prediction of therapy failure to ICB therapy was the shift in the volume to surface ratio between MRI1 and MRI2 (Fig. 2f). As diagnosis, tumor imaging and response monitoring for glioblastoma patient is routinely performed with T1 -weighted contrast-enhanced MRI, response evaluation by T2weighted imaging was validated by simultaneous $\mathrm{T} 1-$ weighted 
a
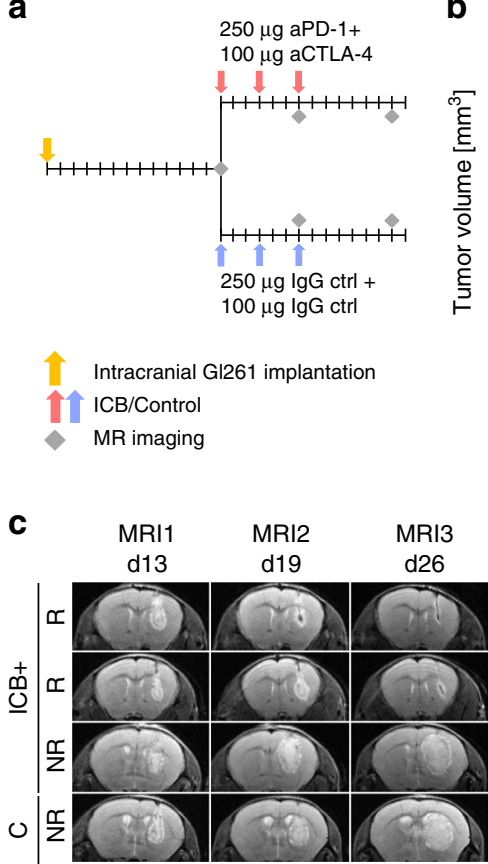

e

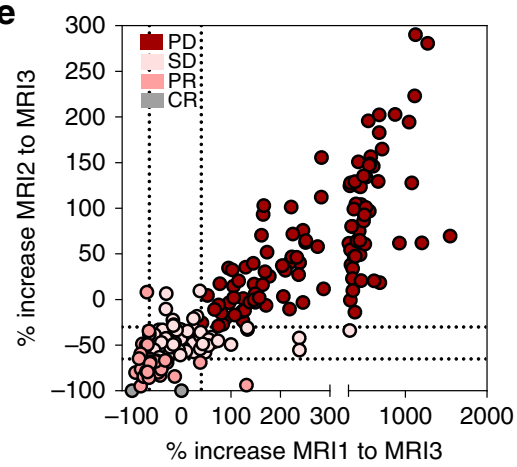

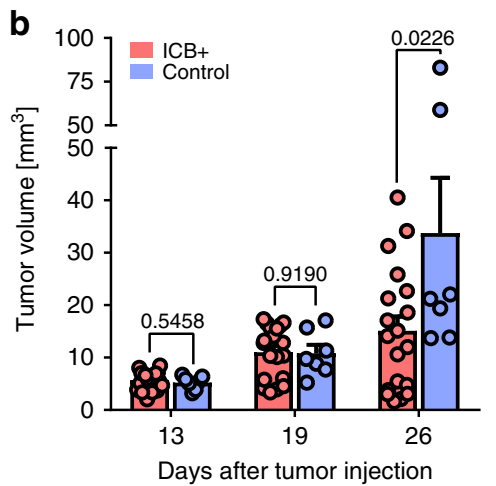

d

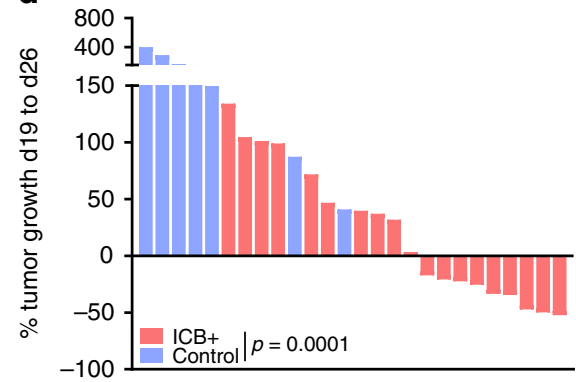

f

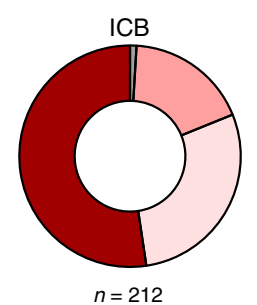

PD $52.36 \%$ | NR $52.36 \%$ SD $28.77 \%$ CR $0.94 \%$
Response ICB vs. C: $p<0.0001$

j

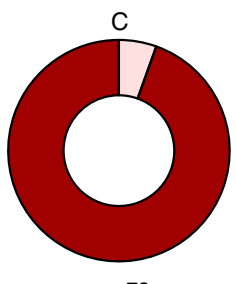

| $5.48 \%$

Mutations
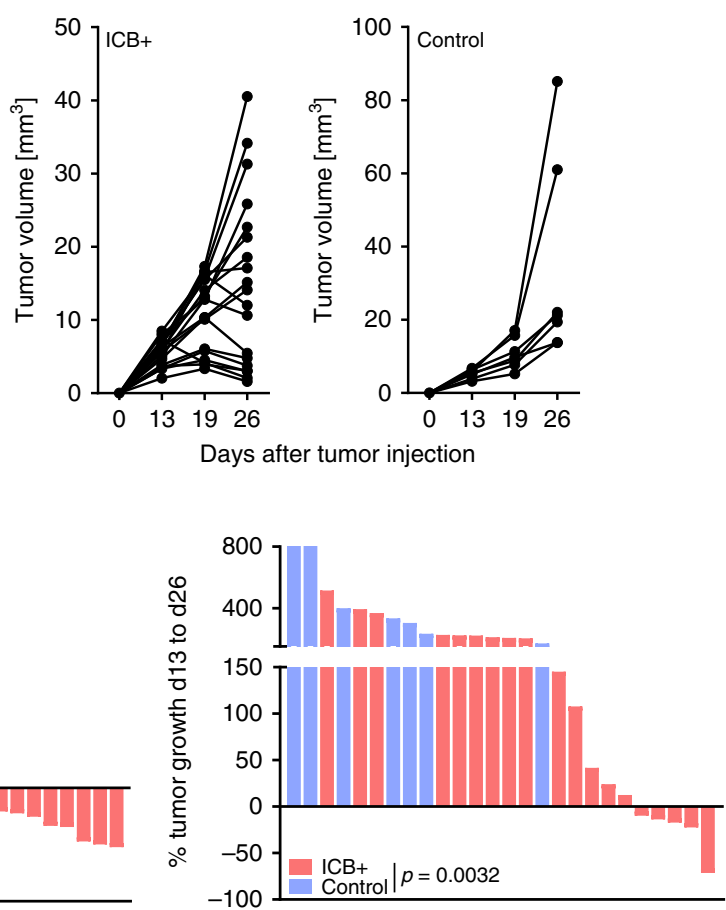

g

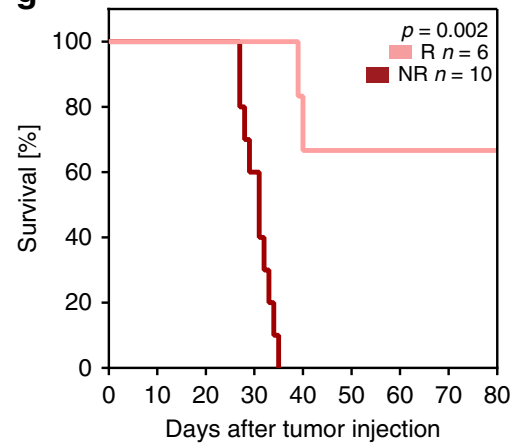

h

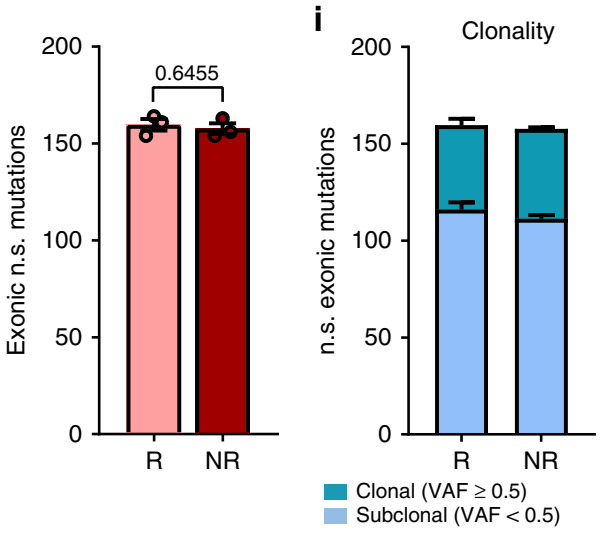

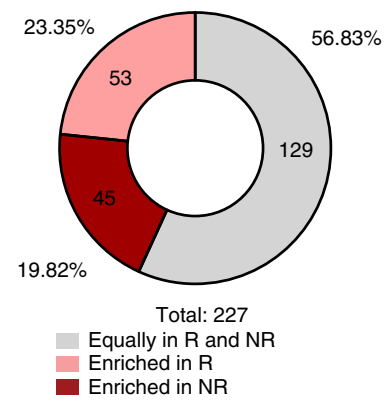

Enriched in R
Enriched in NR

contrast-enhanced (CE) imaging. T2 and T1 CE tumor volumes strongly correlated ( $R^{2}$ 0.96; Supplementary Fig. $\left.3 c\right)$, and T1 CE measurements did not provide an additional benefit for response prediction in pseudoprogressing ICB R (G2 R; Supplementary Fig. 3d).

Impaired antitumor $T$ cell immunity in ICB NR mice. To unravel mechanisms of ICB treatment failure in ICB NR mice, we next examined intratumoral $\mathrm{T}$ cell infiltration and $\mathrm{T}$ cell cytotoxicity in ICB NR tumors. Although T cell infiltration in ICB NR tumors was significantly lower compared to ICB R tumors (Fig. 3a, Supplementary Fig. 4a), no alterations in relative frequencies of $\mathrm{CD}^{+}$and $\mathrm{CD} 4^{+}$tumor-infiltrating lymphocytes (TILs) of ICB R compared to NR mice were observed (Supplementary Fig. 4a). Antitumor TIL responses of ICB NR TILs were diminished as TILs from ICB NR showed an impaired potency to lyse syngeneic glioma cells ex vivo compared to ICB R and control-treated TILs (Fig. 3b). ICB NR CD8 ${ }^{+}$TILs displayed a 
Fig. 1 PD-1 and CTLA-4 blockade decreases GI261 tumor growth in ICB R mice. a-d C57BI/6 J mice were treated with $250 \mu$ anti-PD-1 and $100 \mu$ g antiCTLA-4 (ICB+) or isotype control (C) on d13, d16, and d19. Tumor growth was monitored by MRI on d13 (MRI1), d19 (MRI2), and d26 (MRI3) post intracranial GI261 injection ( $n=19$ vs. $n=7$ animals). b, c Tumor growth and representative MR images of ICB+responder (R), non-responder (NR), and control-treated (C) mice. d Response assessed by \% of tumor growth between d19 and d26, and between d13 and d26 post tumor inoculation.

e, f Advanced response evaluation was performed on an extended dataset (ICB $n=212$ vs. $C n=73$ animals). Mice were grouped according to their response pattern with complete response (CR): \% $\mathrm{V}_{\mathrm{MRI3}-\mathrm{MRI1}}-100 \%$, partial response (PR): \% $\mathrm{V}_{\mathrm{MRI3}-\mathrm{MRI1}} \leq-65.0 \%$ or $\% \mathrm{~V}_{\mathrm{MRI3}-\mathrm{MRI2}} \leq-65.0 \%$, sfig disease (SD): $\% V_{M R I 3-M R I 1}>-65.0 \%$ and $<+40.0 \%$ or $\% V_{M R I 3-M R I 1} \geq+40.0 \%$ and $\% V_{M R I 3-M R I 2} \leq-30 \%$ and progressive disease (PD): $\% V_{M R I 3-M R I 1} \geq$

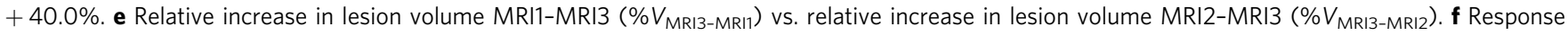
pattern of ICB and C mice. $\mathbf{g}$ Survival of ICB R and ICB NR mice ( $n=6$ vs. $n=10$ animals). Data of two independent experiments were pooled. $\mathbf{h}$ Tumors of ICB R and ICB NR mice were excised on d26 post tumor inoculation and exonic non-synonymous (n.s.) mutational load was assessed by exome sequencing ( $n=3$ vs. $n=3$ animals). $\mathbf{i}, \mathbf{j}$ Clonality of mutations in ICB R and ICB NR tumors $\mathbf{i}$ and mutations predominantly enriched in ICB R or ICB NR tumors, VAF, variant allele frequency. $\mathbf{j}$ Data are represented as mean \pm SEM for $\mathbf{b}, \mathbf{h}$ and $\mathbf{i}$. Statistical significance was determined by two-tailed Student's $t$-test for $\mathbf{b}, \mathbf{d}$ and $\mathbf{h}$, Fisher's exact test for $\mathbf{f}$ or log-rank Mantel-Cox test for $\mathbf{g}$. Source data are provided as a Source Data file.

more polyclonal $\mathrm{T}$ cell receptor (TCR $\beta$ ) repertoire compared to ICB R CD8 ${ }^{+}$TILs, suggesting a failure of proliferation of tumorreactive clones in NR tumors (Fig. 3c). This was further supported by the identification of a shared CDR3 sequence motif (alterations of 1 or less AA between ICB R mice) in the CD8 TIL population of ICB $\mathrm{R}$ mice that was not present in ICB NR CD8 TILs (Supplementary Fig. 4b). Additionally, ICB R tumors were characterized by significantly reduced frequencies of regulatory $\mathrm{T}$ cells $\left(\mathrm{T}_{\text {regs }} ;\right.$ Fig. $3 \mathrm{~d}$ ). No significant evidence of differential PD-1 surface expression or upregulation of immunosuppressive molecules (CD73 and CD38) on $\mathrm{NR} \mathrm{CD}^{+}$and CD8 ${ }^{+}$TILs was observed (Supplementary Fig. 4d, gating strategy Supplementary Fig. 5a, c). In order to assess if responding mice developed longterm immunity against Gl261 cells, Gl261-bearing mice were treated with ICB as previously described, response was assessed by MRI between $\mathrm{d} 21$ and $\mathrm{d} 29$, and mice were followed up for 57 days after tumor inoculation until lesions regressed completely or showed stable, minimal lesion volumes. Responding mice were rechallenged with Gl261 cells by intracranial injection into the contralateral hemisphere at day 57 and were followed for 63 days. Here, rechallenged $\mathrm{R}$ mice did not develop Gl261 tumors as confirmed by MRI and survival analysis (Fig. 3e, f), suggesting an efficient activation of tumor-reactive $\mathrm{T}$ cells and a protective long-term immunity in ICB $\mathrm{R}$ mice. Strikingly, depletion of $\mathrm{CD}^{+} \mathrm{T}$ cells was not sufficient to abrogate response to ICB, while no tumor showed ICB-induced regression after depletion of $\mathrm{CD}^{+} \mathrm{T}$ cells (Fig. 3g, h; Supplementary Fig. 4e-f). This suggests an important role of effector $\mathrm{CD} 4{ }^{+} \mathrm{T}$ cells in driving the response to checkpoint blockade.

Suppressive myeloid cell infiltrates mediate ICB failure. Based on the importance of $\mathrm{CD}^{+} \mathrm{T}$ cells for mediating response to ICB and their close interaction with myeloid cells, we reasoned that antitumor $\mathrm{T}$ cell responses are critically shaped by the intratumoral myeloid compartment interacting with $\mathrm{CD}^{+} \mathrm{T}$ cells. Resistance to ICB therapy in other tumor types has previously been linked to tumor-associated macrophages (TAM) and myeloid-derived suppressor cells ${ }^{17,18}$. Therefore, we investigated the presence and phenotype of glioma-infiltrating myeloid cells in ICB $\mathrm{R}$ and NR mice. $t$ SNE-guided ( $t$-Distributed Stochastic Neighbor Embedding) immune cell subset identification by multiparameter flow cytometry analysis revealed markedly decreased frequencies of tumor-infiltrating myeloid cell subsets, including monocytes, monocyte-derived cells (MDCs), and macrophages in ICB R compared with NR animals (Fig. 4a, gating strategy Supplementary Fig. 5a-d, Supplementary Fig. 6a). Of note, there was no evidence for enhanced apoptosis of CD $45^{\text {high }} \mathrm{CD} 11 \mathrm{~b}^{+}$cells in ICB R tumors (Supplementary Fig. 6b, c). Despite decreased frequencies of tumor-infiltrating myeloid cells in ICB R tumors, we did not observe significant differences in the frequency of circulating blood $\mathrm{CD} 11 \mathrm{~b}^{+}$cells and their expression of the chemokine receptors CCR2, CCR4, CCR5, and CCR6 involved in myeloid cell recruitment to gliomas during ICB therapy (d15; Supplementary Fig. 6d, e) and response establishment (d21; Supplementary Fig. 6f, g). Moreover, cytokine/chemokine array analysis of ICB R, NR, and control-treated mice did not reveal enhanced plasma levels of myeloid cell-attractant chemokines and factors, such as CCL2, CCL3, CCL4, CCL5, CCL11, CCL17, Macrophage colony-stimulating factor (M-CSF) and granulocyte M-CSF in NR plasma during the early treatment phase (Supplementary Fig. 6h).

Targeting of myeloid cells by CSF1R inhibition has been investigated for the treatment of glioblastoma patients with the aim of (1) hindering myeloid cell infiltration into the tumor and (2) reprogramming suppressive myeloid cells to a proinflammatory phenotype ${ }^{19,20}$. Here, we reasoned that CSF1Rtargeted therapy might elevate ICB response by releasing $\mathrm{T}$ cell suppression by intratumoral myeloid cells. CSF1R blockade by monoclonal antibodies increased therapy response from $33.33 \%$ (ICB) to $54.53 \%$ (ICB + CSF1R blockade) with only 2 out of 11 mice showing tumor progression of $>40 \%$ between MRI 2 and MRI3 (compared to 6 out of 12 in the ICB cohort; Fig. 4b, Supplementary Fig. 7a, b).

Glioma-associated myeloid cells have been reported to suppress antitumor $\mathrm{T}$ cell responses and promote tumor progression $^{21}$. We hence sought to characterize the myeloid cell phenotype, and activation in ICB R and NR tumors in more detail. Intratumoral MDCs and macrophages from ICB $\mathrm{R}$ mice expressed higher levels of major histocompatibility complex (MHC) II, while the expression of the immunosuppressive molecules PD-L1 and the poliovirus receptor (CD155) was strongly reduced (Fig. 4c, Supplementary Fig. 8a, b). Expression of PD-L2, the second ligand of PD-1, was not differentially regulated in MDCs and macrophages in ICB R compared to NR tumors (Supplementary Fig. 8b). PD-L1 in the tumor microenvironment was predominantly expressed on intratumoral monocytes, MDCs, and macrophages, while expression of PDL2 was less restricted and present on other immune cell subsets as well as tumor and stroma cells (Fig. 4d). Moreover, MDCs in ICB $\mathrm{R}$ tumors showed increased levels of tumor necrosis factor (Fig. 4c, Supplementary Fig. 8a), suggesting a pro-inflammatory phenotype. In line with these findings, NanoString gene expression analysis of intratumoral $\mathrm{CD} 45^{\text {high }} \mathrm{CD} 11 \mathrm{~b}^{+}$cells revealed that $\mathrm{CD} 45^{\text {high }} \mathrm{CD} 11 \mathrm{~b}^{+}$cells from ICB NR tumors displayed an increased expression of anti-inflammatory genes involved in inhibition of IL1b- and IL1a-mediated inflammatory responses (Illrn), IL4 signaling (IL4ra), and M2-associated genes, such as $P D-L 1, T G F b i$, and the scavenger receptor Msr1 (Fig. 4e, f). Contrary, CD $45^{\text {high }} \mathrm{CD} 11 \mathrm{~b}^{+}$cells from ICB R tumors 
a

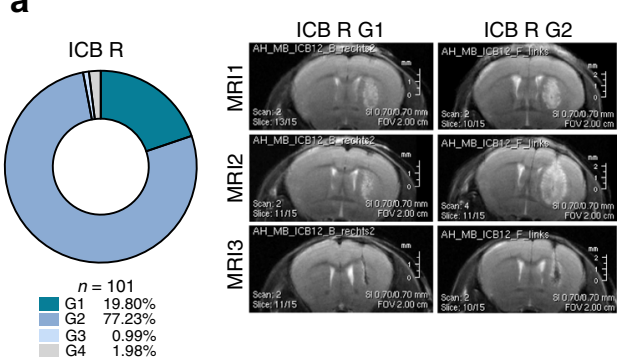

b

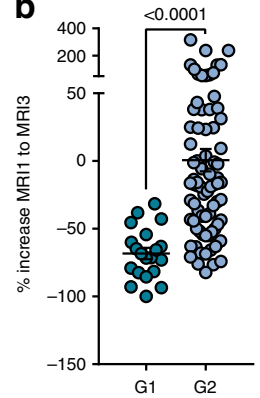

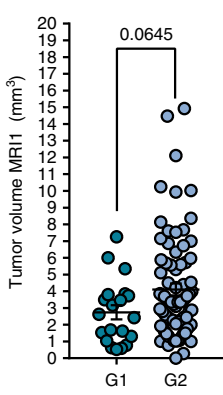

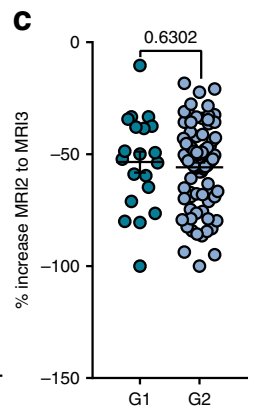

d

e
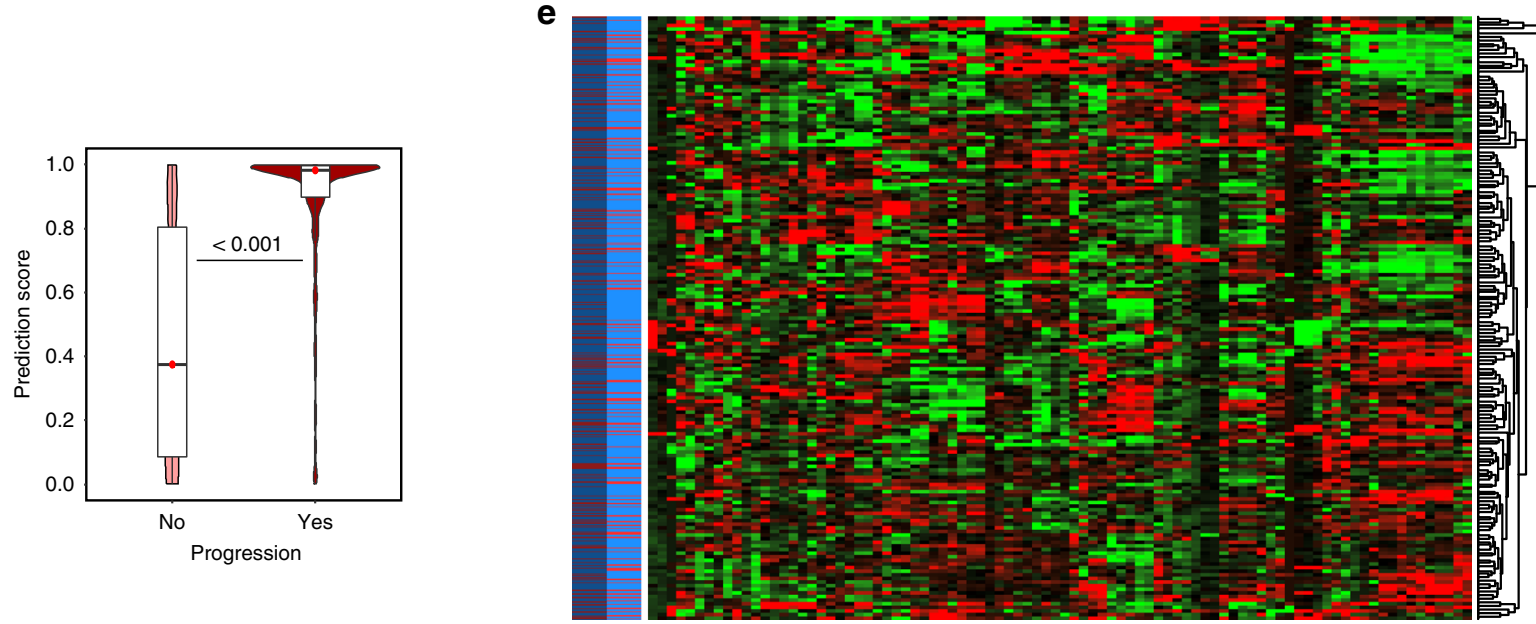

Radiomic prediction

Ground truth

No PD

PD

No PD

PD

\begin{tabular}{|c|c|c|}
\hline Rank & Timepoint & Feature \\
\hline & Difference MRI1/MRI2 & Volumetric \\
\hline 2 & MRI1 & Co-occurenced based \\
\hline 3 & Difference MRI1/MRI2 & Co-occurenced based \\
\hline 4 & MRI1 & Co-occurenced based \\
\hline 5 & Difference MRI1/MRI2 & Volumetric \\
\hline 6 & Difference MRI1/MRI2 & Curvature \\
\hline 7 & Difference MRI1/MRI2 & Neighbourhood grey tone difference \\
\hline 8 & Difference MRI1/MRI2 & Co-occurenced based \\
\hline 9 & Difference MRI1/MRI2 & Volumetric \\
\hline 10 & Difference MRI1/MRI2 & Neighbouring grey level dependence \\
\hline 11 & Difference MRI1/MRI2 & Co-occurenced based \\
\hline 12 & Difference MRI1/MRI2 & Volumetric \\
\hline 13 & Difference MRI1/MRI2 & Co-occurenced based \\
\hline 14 & MRI1 & Curvature \\
\hline 15 & Difference MRI1/MRI2 & Skewness \\
\hline 16 & Difference MRI1/MRI2 & Curvature \\
\hline 17 & Difference MRI1/MRI2 & Curvature \\
\hline 18 & Difference MRI1/MRI2 & Run length \\
\hline 19 & Difference MRI1/MRI2 & Co-occurenced based \\
\hline 20 & Difference MRI1/MRI2 & Neighbouring grey level dependence \\
\hline
\end{tabular}

Parameter

Surface to volume ratio - mesh based

Bins 128 fullimage range 1 - Std.Dev. joint average

1 - Std.Dev. joint variance Compactness 1 old - mesh based

Std.Dev. negative mean curvature

Bins 128 fullimage range 1 - Std. Dev. joint averag PCA major axis length

Bins 128 fullimage range 1 - average neighbourhood size Bins 128 fullimage range 1 - Std.Dev. correlation Surface - mesh based

Bins 128 fullimage range 1 - over all difference entropy Negative minimum curvature

Negative maximum curvature

Negative mean curvature

Mean curvature

Bins 128 fullimage - high grey level run emphasis Std. Bins 128 fullimage range 1 - Std.Dev. joint maximum Bins 128 fullimage range 1 - average incomplete Neighbourhood size

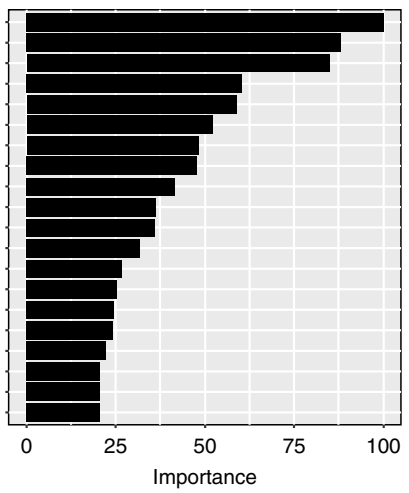

Fig. 2 Radiomic prediction of therapy response to ICB therapy. a-c C57BI/6 J mice were treated with $250 \mu$ anti-PD-1 and $100 \mu \mathrm{g}$ anti-CTLA-4 (ICB + ) and tumor growth, therapy response and pseudoprogression were evaluated by MRI before (MRI1), during (MRI2), and after ICB therapy (MRI3). a Growth pattern analysis of ICB R $\left(n=101\right.$ animals). G1: $\% V_{\text {MRI2-MRI1 }}<0 \%$ and $\% V_{\text {MRI3-MRI2 }}<0 \%$; G2: $\% V_{\text {MRI2-MRI1 }}>0 \%$ and $\% V_{\text {MRI3-MRI2 }}<0 \%$; G3: \% $V_{\text {MRI2-MRI1 }}$ $>0 \%$ and $\% V_{M R I 3-M R I 2}>0 \%$; and G4: $\% V_{M R I 2-M R I 1}<0 \%$ and $\% V_{M R I 3-M R I 2}>0 \%$. b $\% V_{M R I 3-M R I 1}$ (left), $V_{M R I 1}$ (baseline tumor volume; middle), and $V_{M R I 3}$

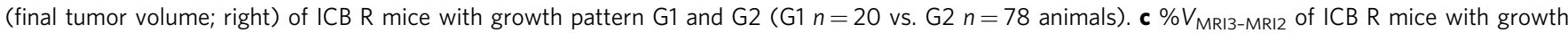
pattern $\mathrm{G} 1$ and $\mathrm{G} 2$ ( $\mathrm{G} 1 n=20$ vs. $\mathrm{G} 2 n=78$ animals). d-f Radiomic response prediction after ICB therapy based on radiomic features of MRI1 (baseline) and MRI2 (during ICB therapy) images ( $n=148$ animals). Boxplot with blocks showing the interquartile range (IQR) of data points and horizontal central line (red dot) corresponding to the median. The superimposed violin plot visualizes the distribution of the data and its probability density. Radiomic signature score $\mathbf{d}$, heatmap of radiomic features $\mathbf{e}$, and top predictive radiomic features $\mathbf{f}$ of $\mathrm{R}$ and NR tumors based on radiomic features of MRI1 and MRI2. Data are presented as mean \pm SEM for $\mathbf{b}$ and $\mathbf{c}$. Statistical significance was determined by two-tailed Student's $t$-test for $\mathbf{b}$-d. Source data are provided as a Source Data file.

upregulated pro-inflammatory genes known to induce Th1 T cell responses, including the cytokine Il12 and genes involved in MHC II presentation (H2-DMb2). As PD-L1 was differently expressed on the majority of myeloid cell populations with the strongest differences on intratumoral macrophages (Fig. 4c, Supplementary Fig. 8a), we sought to investigate the impact of
PD-L1 expression on the effector functions of ICB R and NR tumor-associated myeloid cells. Interestingly, differences in PDL1 expression between ICB R and NR were exclusively observed in myeloid cells in the tumor microenvironment and not present in the periphery (Supplementary Fig. 8c). Strikingly, $\mathrm{PD}-\mathrm{L} 1$ expression on intratumoral $\mathrm{CD} 11 \mathrm{~b}^{+}$myeloid cells showed 
a

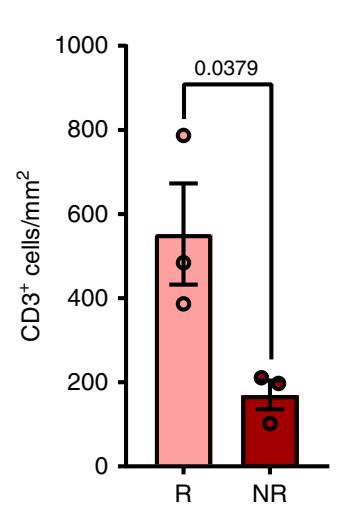

d

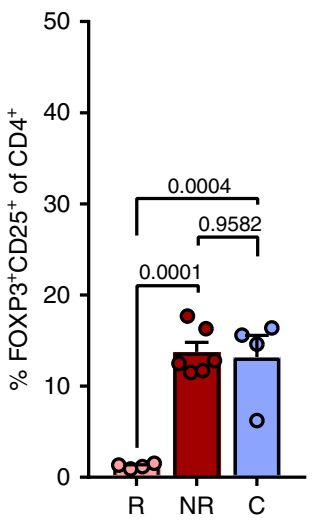

b

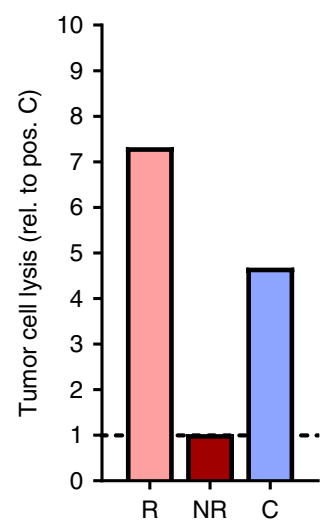

C

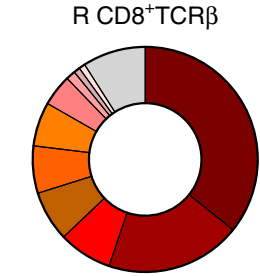

$\%$ of all productive frequencies

C AS SL LA YEQYF

CASSGRD SQNTLYF

C AS SLEP GADT GQ LYF

C TCSADRTG GVYAEQFF

C AS SQ DP GP YAEQ FF

C AS SP PG QG RGEQ YF

C AS SALGSQNTLYF

CASSL GRRS GNTL YF

C AS SQDLNS DYTF

C AS SPRGVQDT QYF

Others

NR CD8 ${ }^{+}$TCR $\beta$

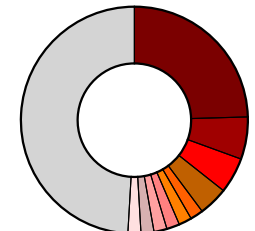

$\%$ of all productive frequencies

CASSRVGDTQYF

C ASSLTG VNSDYTF

C GARE GG GYEQ YF

C AS SS TG AN SDYT

CAS SLRE QYF

CASSDAGTGEDTQYF

CASSRDKAINQDT QYF

CASSRGRGR SYEQYF

C AS SF GPEYEQYF

CASSLFRPNSDYTF

Others

e

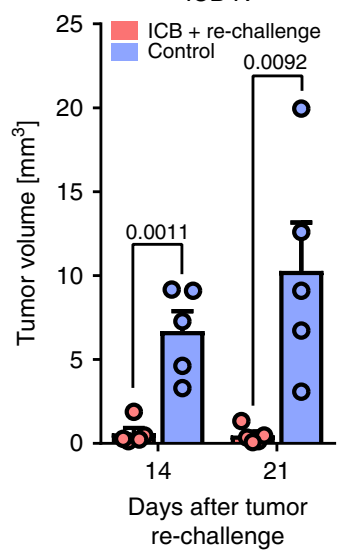

f
ICB R

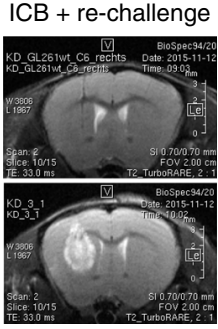

Control

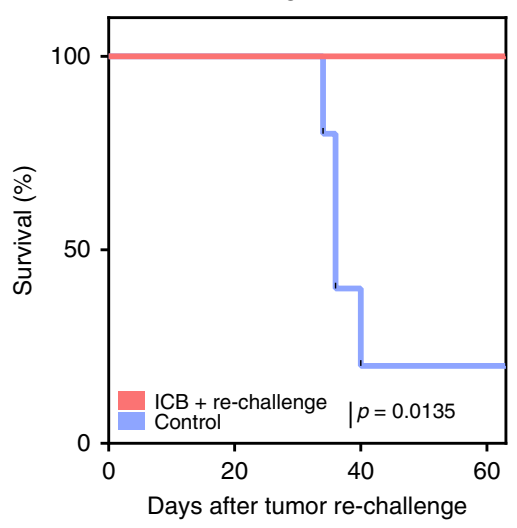

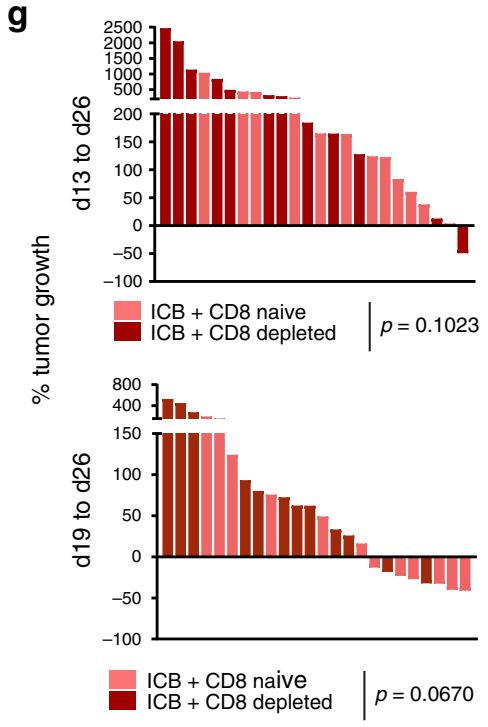

g

a strong negative correlation with response to ICB therapy (Supplementary Fig. 9a). The immunosuppressive molecule PD$\mathrm{L} 1$ is known to inhibit $\mathrm{T}$ cell proliferation and effector function upon binding to its ligand PD-1 on T cells ${ }^{1}$. As PD- 1 was blocked in ICB-treated mice, we investigated whether PD-L1/PD-1 signaling directly impacts macrophage activation and function, such as phagocytosis as previously proposed ${ }^{22,23}$. Here, we did not observe an increased phagocytotic activity of tumor-associated $\mathrm{CD} 45^{\text {high }} \mathrm{CD} 11 \mathrm{~b}^{+}$cells isolated from ICB $\mathrm{R}$ compared to ICB h

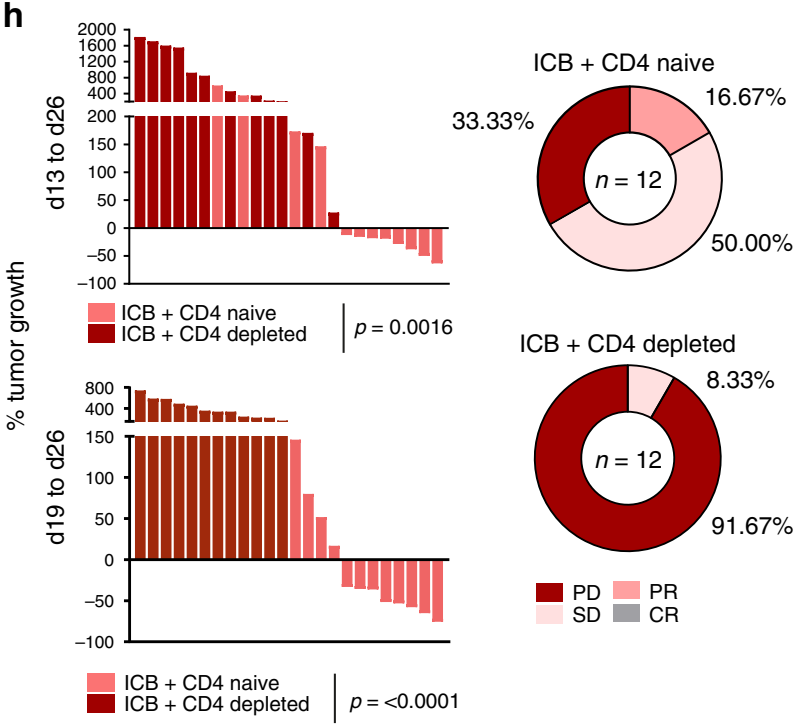

NR mice (Supplementary Fig. 9b). Moreover, ex vivo PD-L1 inhibition during phagocytosis did not induce enhanced phagocytic activity of TAM (Supplementary Fig. 9b). Notably, CD11b ${ }^{+}$ PD-L1 ${ }^{-}$cells in the tumor microenvironment of ICB R mice showed increased expression of MHC II compared to NR PD-L1- myeloid cells, suggesting enhanced antigen presentation by this PD-L1 ${ }^{-}$myeloid cell subset (Supplementary Fig. 9c). To rule out that increased frequencies of suppressive myeloid cells in ICB NR tumors are a result of increased tumor size independent 
Fig. 3 Impaired antitumor $\mathbf{T}$ cell immunity in ICB NR tumors. C57BI/6 J mice were treated with $250 \mu \mathrm{g}$ anti-PD-1 and $100 \mu \mathrm{g}$ anti-CTLA-4 (ICB + ), or isotype control (C) on $\mathrm{d} 13, \mathrm{~d} 16$, and $\mathrm{d} 19$ and tumor monitoring was performed on $\mathrm{d} 13, \mathrm{~d} 19$, and $\mathrm{d} 26$ post tumor inoculation. $\mathbf{a}$ CD3 ${ }^{+}$cell counts per mm ${ }^{2}$ tumor area assessed by immunohistochemistry (ICB R $n=3$, ICB NR $n=3$ animals). b CD3 ${ }^{+}$TILs were isolated by MACS from ICB R, ICB NR, and C tumors on $\mathrm{d} 27$ and incubated for $4 \mathrm{~h}$ with Gl261 cells ex vivo. Cytotoxicity was analyzed by LDH release relative to positive lysis control (ratio). Five samples per group were pooled. Values are corrected for spontaneous effector and target cell LDH release. c Representative ICB R and ICB NR CD8 ${ }^{+}$TCR $\beta$ TIL repertoire and $\%$ of ten most frequent sequences. $\mathbf{d}$ Flow cytometry for frequency of CD25+FOXP3 ${ }^{+} \mathrm{T}_{\text {regs }}$ of CD4+ TILs $(I C B R n=4$, ICB NR $n=6, C$ $n=4$ animals). e, $f$ C57BL/6 J mice were treated with ICB on d14, d17, and d20 after GI261 injection and tumors were measured on d14, d21, d29, d42, and d50. GI261 rechallenge of ICB R was performed on d57 after first tumor injection. Tumor volumes on d14 and d21 after rechallenge e and survival $\mathbf{f}$ of GI261 rechallenged ICB R and control-injected mice ( $n=5$ vs. $n=5$ animals). $\mathbf{g}, \mathbf{h}$ CD8 ${ }^{+}$or $C D 4^{+}$T cells were depleted prior and during ICB using monoclonal depletion antibodies $\left(4 \times 500 \mu \mathrm{g} 2.43\right.$ or $2 \times 1000 \mu \mathrm{g}$ GK1.5). $\mathbf{g}$ ICB response in CD8 ${ }^{+}$-depleted or naive mice $(\mathrm{ICB}+\mathrm{CD} 8$ naive $n=13, \mathrm{ICB}+\mathrm{CD} 8$ depl. $n=13$ animals) and $\mathbf{h}$ in CD4 depleted or naive mice (ICB + CD4 naive $n=12, I C B+C D 4$ depl. $n=12$ animals). Data are represented as mean \pm SEM for $\mathbf{a}, \mathbf{d}$ and $\mathbf{e}$. Statistical significance was determined by one-way ANOVA with Tukey's test for $\mathbf{d}$, two-tailed Student's t-test for $\mathbf{a}, \mathbf{e}, \mathbf{g}$ and $\mathbf{h}$ or log-rank Mantel-Cox test for $\mathbf{f}$. Source data are provided as a Source Data file.

of treatment rather than a mechanism of ICB resistance, we correlated MRI3 tumor volumes with frequencies of tumorassociated $\mathrm{CD} 45^{\text {high }} \mathrm{CD} 11 \mathrm{~b}^{+}$cells and percentage of $\mathrm{PD}-\mathrm{L} 1^{+}$cells on tumor-associated $\mathrm{CD} 45^{\text {high }} \mathrm{CD} 11 \mathrm{~b}^{+}$cells in ICB and C-treated mice. While MRI3 tumor volumes strongly correlated with both factors in ICB mice, no correlation was observed in $\mathrm{C}$ mice (Supplementary Fig. 10a-c, left panel). Moreover, frequencies of tumor-associated $\mathrm{CD} 45^{\text {high }} \mathrm{CD} 11 \mathrm{~b}^{+}$cells and percentage of $\mathrm{PD}-\mathrm{L} 1{ }^{+}$cells on tumor-associated $\mathrm{CD} 45^{\text {high }} \mathrm{CD} 11 \mathrm{~b}^{+}$cells were significantly correlated with tumor growth (MRI1 to MRI3, and MRI2 to MRI3) in ICB but not C mice (Supplementary Fig. 10a-c, middle (MRI1 to MRI3) and right (MRI2 to MRI3) panel). Heterogeneity of tumor volumes in $\mathrm{C}$ mice does not reflect a heterogeneity in the suppressive CD11b compartment.

$\mathrm{CD4}^{+}$TIL suppression by the PD-L1/PD-1/CD80 axis. To address whether of PD-L1-expressing myeloid cells directly impact $\mathrm{T}$ cell activation and proliferation in the tumor microenvironment, we analyzed the ability of macrophages isolated from ICB R, NR, and control tumors to suppress T cell proliferation and effector function. Suppression of $\mathrm{CD}^{+}$, but not $\mathrm{CD}^{+} \mathrm{T}$ cell proliferation was more pronounced when $\mathrm{T}$ cells were co-cultured with tumor-associated myeloid cells from ICB NR compared to R (Fig. 5a, Supplementary Fig. 11a-c). This was accompanied by an increased expansion of $\mathrm{T}_{\text {regs }}$ after co-culture with tumor-associated myeloid cells from ICB NR (Supplementary Fig. 11d). Furthermore, $\mathrm{CD}^{+} \mathrm{T}$ cell suppression and $\mathrm{T}_{\text {reg }}$ expansion were reduced upon PD-L1 inhibition during co-culture with ICB NR tumor-associated myeloid cells (Fig. 5a, Supplementary Fig. 11c, d). As PD-1 is blocked by the ICB regimen used here, we hypothesized that $\mathrm{T}$ cell suppression is established by an alternative binding partner of PD-L1 on T cells. Interestingly, CD80 has been proposed to act as an alternative binding partner of PD-L1 on T cells, thereby suppressing $\mathrm{T}$ cell proliferation and activation $^{24}$. Indeed, we confirmed CD80 expression on CD4 ${ }^{+}$ and $\mathrm{CD}^{+}{ }^{+} \mathrm{T}$ cells of ICB-treated mice, with a predominant expression in the TIL compartment (Fig. 5b, Supplementary Fig. 11e). Moreover, CD80 expression on naive, pre-activated $\mathrm{T}$ cells was induced upon co-culture with tumor-associated myeloid cells and levels of CD80 positive CD4 T cells after coculture with tumor-associated myeloid cells was comparable to levels on CD4 TILs (Fig. 5c, Supplementary Fig. 11e). To investigate if blockade of the PD-L1/CD80 interaction can restore response to anti-PD-1 + anti-CTLA-4 therapy, PD-L1 blocking antibodies were administered in addition to the anti-PD-1 and anti-CTLA-4 regimen. Triple ICB resulted in a decreased tumor growth and enhanced response $(11 / 13$ vs. $6 / 13)$ to ICB therapy when compared with ICB targeting PD-1 and CTLA-4 only (Fig. 5d, Supplementary Fig. 11f). To confirm these preclinical findings, we evaluated macrophage frequencies in glioblastoma patients from a recently published clinical trial of anti-PD-1 treatment ${ }^{25}$. From this dataset, we applied CIBERSORT analysis of RNA sequencing data from GBM tissue of $\mathrm{R}(n=4)$ and NR $(n=5)$ patients before anti-PD-1 therapy. In line with our findings, intratumoral M2 macrophage levels as well as myeloid cell infiltrate levels (monocytes, M0, M1, and M2 macrophages) showed a trend toward elevated levels in NR glioblastoma patients before anti-PD-1 therapy (Fig. 5e), supporting the hypothesis that suppressive myeloid cell subsets impair the induction of antitumor $\mathrm{T}$ cell responses by ICB therapy. In summary, our findings suggest a distinct set of biomarkers associated with response to ICB in a hypermutated syngeneic glioma model that is dominated by innate (absence of intratumoral macrophages and absence of PD-L1 on intratumoral macrophages) rather than adaptive immune parameters (Fig. 5f).

\section{Discussion}

Immune checkpoint inhibitors for glioma patients are now tested in clinical trials and first results point toward poor responses, although neo-adjuvant ICB therapy was recently shown to promote a survival benefit in glioblastoma patients $6,26,27$. These studies indicate that mechanism-driven response biomarkers and combination strategies are required, in order to define which patients benefit from checkpoint therapy and to simultaneously enhance therapy response.

Preclinical models have been notoriously problematic for the identification of biomarkers, not only as they incompletely reflect tumor biology but also because they seemingly lack interindividual heterogeneity. We have uncovered and mechanistically dissected the surprising finding that heterogeneity of response is not only observed in humans but also in syngeneic tumor models in inbred mice. This observation may offer the opportunity to not only elucidate novel mechanisms of response and resistance and, novel therapeutic targets suitable for combination therapies, but also identify potential predictive biomarkers potentially applicable to patients with gliomas (Fig. 5f). We have utilized this robust heterogeneity of response and resistance with pseudoprogression, signifying an immune response in a syngeneic highmutational load experimental glioma model to establish a radiomic-based MRI signature, predicting response with high accuracy. This signature may be useful for future clinical trials enriched for patients with hypermutated glioblastoma similar to the experimental model used here. Proposed signature might additionally be applied in combination with automated quantitative tumor response assessment of MRI, using artificial networks that will allow for improved clinical decision making 28 . The establishment and application of additional MR protocols to 

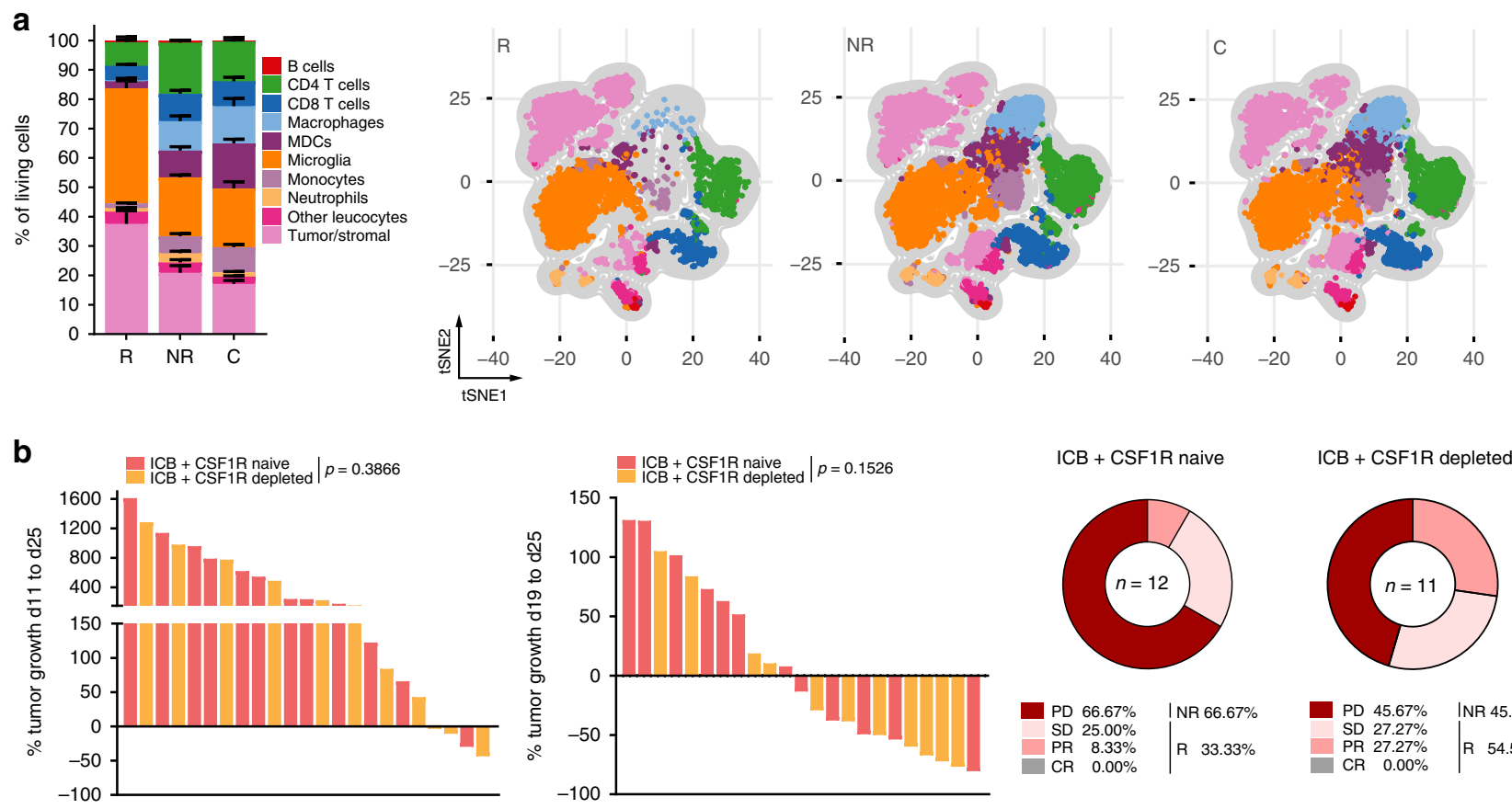

c
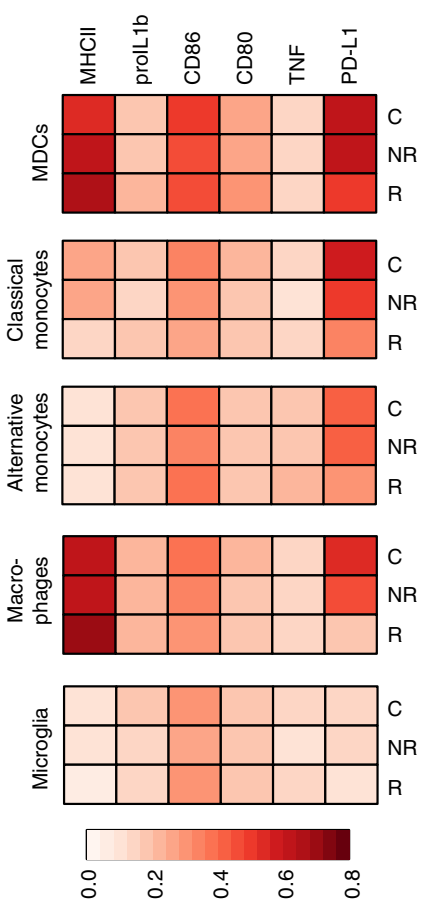

d

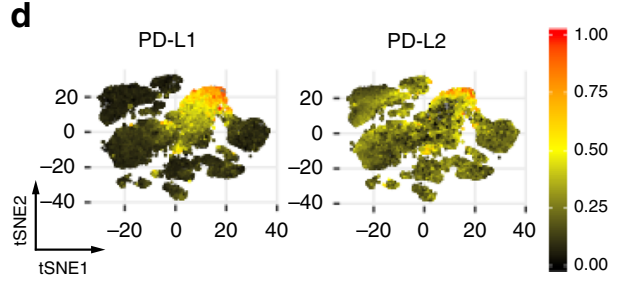

e

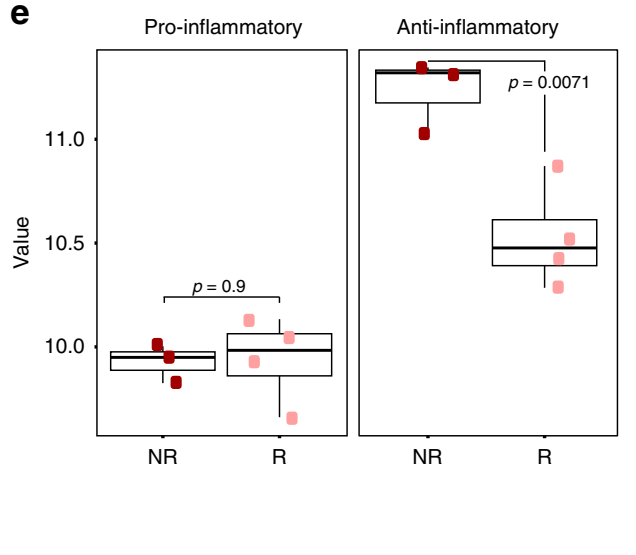

f
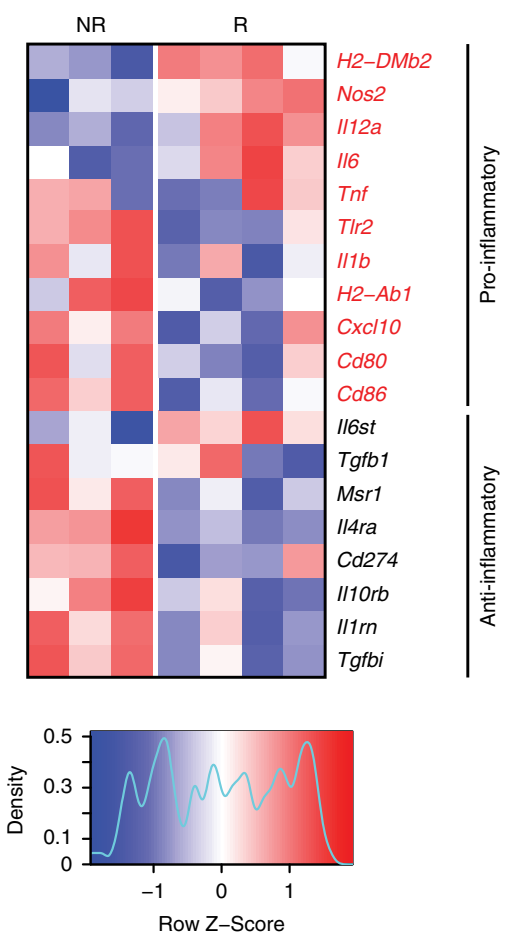

Fig. 4 Enhanced frequencies of PD-L1-expressing macrophages in ICB NR tumors. C57BI/ $6 \mathrm{~J}$ mice were treated with $250 \mu \mathrm{g}$ anti-PD-1 and $100 \mu \mathrm{g}$ antiCTLA-4 (ICB + ), or isotype control (C) on d13, d16, and d19 and tumors were monitored by MRI on d13, d19, and d26 post GI261 injection. a Multiparameter flow cytometry analysis of CNS samples from ICB R, ICB NR, and C on d27. (ICB R $n=5, I C B N R n=5, C n=5$ animals). tSNE-guided immune cell subset identification using tSNE composite dimensions by multiparameter flow cytometry analysis. Relative frequencies (left) and FlowSOMguided meta-clustering on living and single cells (right) of ICB R, NR, and C CNS tissue. b CSF1R was targeted prior and during ICB therapy using monoclonal antibodies (AFS98; $6 \times 250 \mu \mathrm{g}$ ). Response to ICB therapy in CSF1R-targeted and control mice $(I C B+n=12, I C B+C S F 1 R$ depleted $n=11$ animals). c Multiparameter flow cytometry analysis of CNS samples from ICB R, ICB NR, and C mice on d27. (ICB R $n=5, I C B N R n=5, C n=5$ animals). Heatmaps showing the median expression (value range $0-1$, white-red) of pro- and anti-inflammatory markers in MDCs, classical monocytes, alternative monocytes, macrophages, and microglia clusters in ICB R, NR, and C CNS tissue. d PD-L1 and PD-L2 expression on identified CNS subsets from stochastically selected cells from ICB R, ICB NR, and C CNS tissue. e, $\mathbf{f}$ Pro- and anti-inflammatory gene signature score (geometric mean of pro- and antiinflammatory genes) $\mathbf{e}$ and gene expression of pro- and anti-inflammatory genes $\mathbf{f}$ in tumor-associated CD45 high CD11b ${ }^{+}$cells (macrophages) from ICB $R$ and ICB NR assessed by NanoString analysis (ICB R $n=4, I C B N R n=3$ animals). Center line of the boxplot shows the mean and the whiskers represent the upper and lower most quartiles. Data are represented as mean \pm SEM for a. Statistical significance was determined by two-tailed Student's $t$-test for b and e. Source data are provided as a Source Data file. 
a
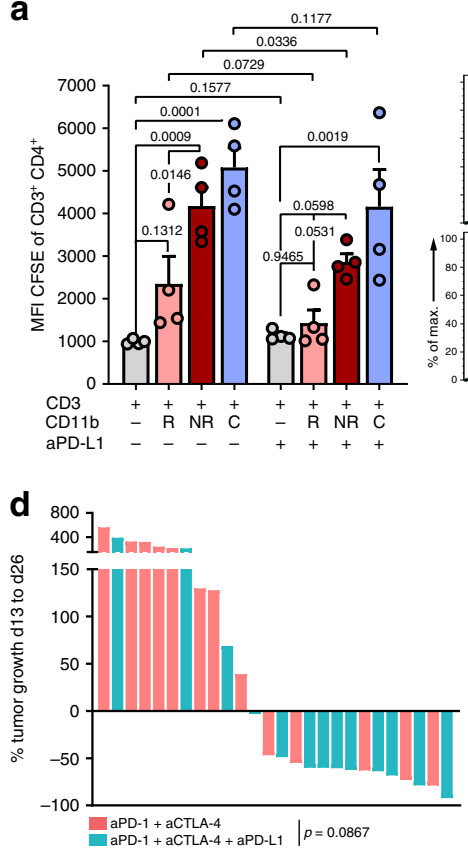

e

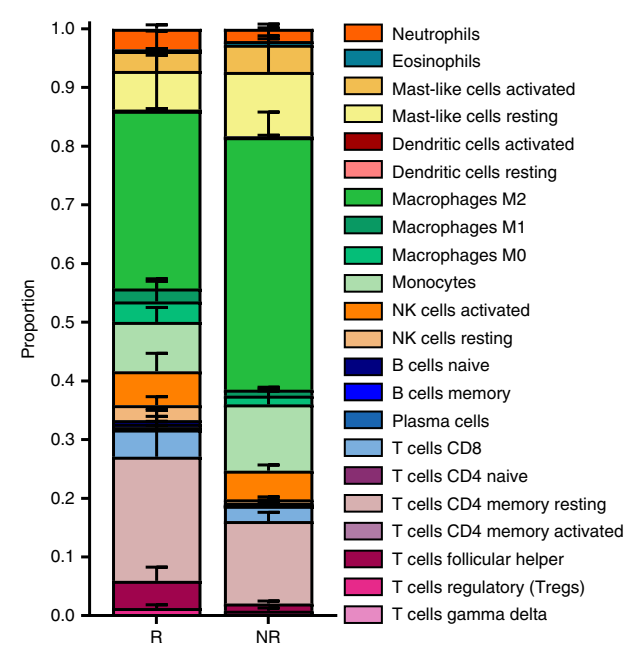

R N b
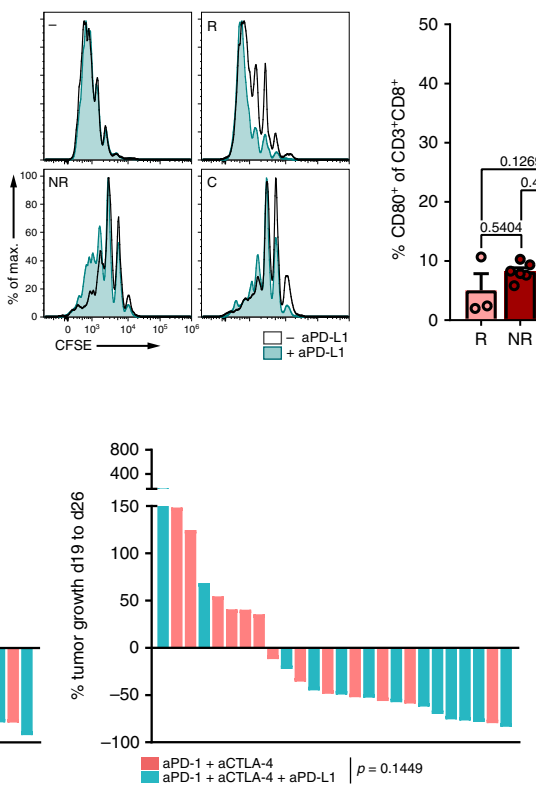
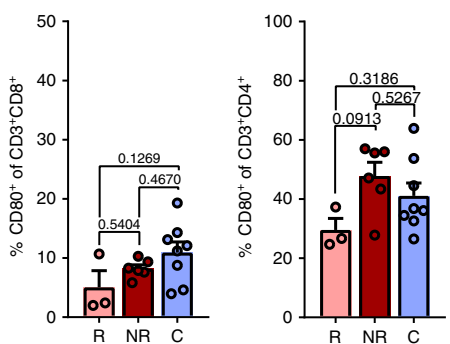

c
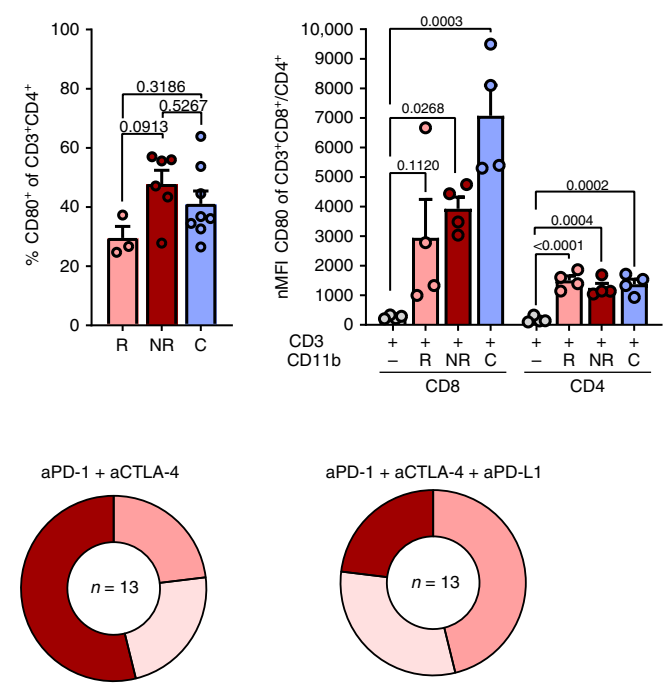

PD $53.85 \%$ | NR $53.85 \%$ \begin{tabular}{l|l} 
SD $23.08 \%$ & \\
PR $23.08 \%$ & R $46.16 \%$
\end{tabular} PR $23.08 \%$
CR $0.00 \%$

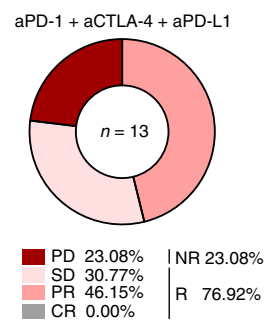

f

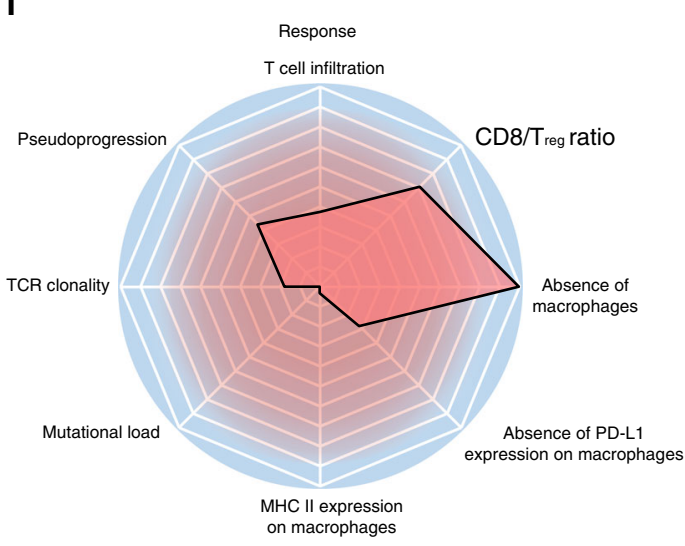

Fig. 5 TAMs establish ICB resistance through PD-L1-CD80-mediated CD4 ${ }^{+} \mathbf{T}$ cell suppression and $\mathbf{T}_{\text {reg }}$ expansion. $\mathbf{a}$, $\mathbf{c}$ Ex vivo $T$ cell suppression by tumor-associated myeloid cells. CD11b+ cells were purified from ICB R, ICB NR, and $\mathrm{C}$ tumors on $\mathrm{d} 27$ by MACS and co-cultured for $72 \mathrm{~h}$ with pre-activated

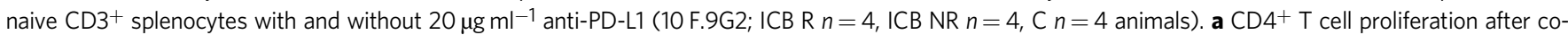
culture assessed by CFSE staining. b Frequency of $\mathrm{CD} 80^{+}$cells of $\mathrm{CD} 8^{+}$(left) and CD4+ (right) TILs (ICB R $n=3, \mathrm{ICB} N R n=6, \mathrm{C} n=8$ animals). c CD80 expression on pre-activated naive $\mathrm{CD} 4^{+}$and $\mathrm{CD} 8^{+} \mathrm{T}$ cells before and after co-culture with tumor-associated myeloid cells from ICB R, ICB NR, and C. $\mathbf{d}$ Tumor growth (left) and response (right) of C57BL/6 J mice treated with $250 \mu \mathrm{g}$ anti-PD-1 and $100 \mu \mathrm{g}$ anti-CTLA-4, and as combinatory therapy with additional $200 \mu \mathrm{g}$ anti-PD-L1 on d13, d16, and d19 post GI261 inoculation (aPD- $1+\mathrm{aCTLA}-4 n=13$, aPD- $1+\mathrm{aCTLA}-4+$ aPD-L1 $n=13$ animals). e CIBERSORT analysis of a GBM expression dataset of PD-1 inhibitor-treated patients before therapy ( $n=4, N R n=5$ biologically independent samples)-two-sided WRST.

f Mediators of ICB response (Z-transformed log2 fold change R/NR). Data are represented as mean \pm SEM for $\mathbf{a}, \mathbf{b}$, $\mathbf{c}$ and $\mathbf{e}$. For $\mathbf{a}$, statistical significance was determined by one-way ANOVA in combination with Dunnett's test $\left(C D 3^{+}\right.$cells $+R$, NR, or $C \mathrm{CD}_{11 b^{+}}$cells vs. T cells only) and Sidak's test for multiple comparison $\left(\mathrm{CD}^{+}\right.$cells $+\mathrm{R} \mathrm{CD11b}{ }^{+}$cells vs. CD3 ${ }^{+}$cells + NR CD11b ${ }^{+}$cells) or two-tailed paired Student's t-test (- PD-L1 vs. + PD-L1). Statistical significance was analyzed by one-way ANOVA with Tukey's test for multiple comparison for $\mathbf{b}$, by one-way ANOVA in combination with Dunnett's test (CD3+ cells $+R$, $\mathrm{NR}$, or C CD11b+ cells vs. T cells only) for $\mathbf{c}$, by unpaired two-tailed Student's $t$-test for $\mathbf{d}$, and WRST with Benjamini-Hochberg correction for $\mathbf{e}$. Source data are provided as a Source Data file.

image immunotherapy-induced immune responses in the CNS will further facilitate therapy monitoring and response evaluation.

Heterogeneity of response allowed for the precise analysis of the glioma immune microenvironment associated with response and resistance in this model. We identified a PD-L1 ${ }^{+}$macrophage subset that drives resistance to ICB by suppression of $\mathrm{CD}^{+} \mathrm{T}$ cell activation and proliferation and $\mathrm{T}_{\text {reg }}$ induction
(Fig. 5a, Supplementary Fig. 11c, d). PD-L1 expression of tumors has long been believed to be the major prerequisite for efficient PD-1 blockade. High PD-L1 expression in the initial tumor tissue was associated with poor response to nivolumab in the CheckMate 143 trial (NCT02017717) for patients with recurrent glioblastoma $^{6}$. Loss of PTEN has been associated with an increase of PD-L1 on glioblastoma cells and immune resistance ${ }^{29-31}$ and in 
clinical trials resistance to $\mathrm{PD}-1$ inhibitors is associated with genetic alterations in the PTEN gene ${ }^{25}$. However, PD-L1 is not only expressed on tumor cells but also infiltrating leukocytes in glioblastoma $^{32-34}$ and PD-L1 expression on macrophages has been associated with poor survival and resistance to immunotherapy for patients with glioblastoma ${ }^{35,36}$. Recent studies highlight the impact of tumor-derived factors on PD-L1 expression on macrophages ${ }^{37-39}$. These clinical studies and our data are in line with previous observations that macrophage infiltration and PD-L1 expression on infiltrating macrophages are critical determinants of resistance to ICB. It is a widely accepted view that signaling induced by PD-1/PD-L1 interaction impairs $\mathrm{T}$ cell effector function and proliferation that subsequently results in $\mathrm{T}$ cell exhaustion and decreased tumor immunity ${ }^{1}$. However, the impact of PD-1/PD-L1 binding on the phenotype, and function of $\mathrm{PD}^{-} 1^{+}$antigen-presenting cells and tumor cells is still incompletely understood. Here, we have shown that PD-L1 expression on macrophages is accompanied by the expression of other immunosuppressive molecules such as CD155 (Supplementary Fig. $8 \mathrm{~b})$ and that frequency of PD-L1 ${ }^{+}$myeloid cells is negatively correlated to therapy response to checkpoint blockade (Supplementary Fig. 9a). This data is supported by evidence that suggests an induction of a regulatory macrophage profile of $\mathrm{PD}-\mathrm{L}^{+}$ macrophages upon PD-L1 signaling ${ }^{40}$. Binding of PD- 1 on T cells to PD-L1 on macrophages hence decreases inflammatory mediators while increasing the production of anti-inflammatory cytokines $^{40}$. It remains to be investigated, if this also holds true for the CD80/PD-L1 interaction. We further hypothesized that PD-1/PD-L1 signaling might interfere with macrophage effector function, such as phagocytosis. Interestingly, PD-1 expression on TAMs has been reported to increase during tumor progression in murine and human tumors, and to negatively correlate with phagocytic potency against tumor cells ${ }^{22}$. In our hands, gliomaassociated macrophages did not induce phagocytosis upon PD-L1 inhibition, suggesting an alternative mechanism of immunosuppression by $\mathrm{PD}-\mathrm{L}^{+}$macrophages. Here, we show that combination of PD-1, CTLA-4, and PD-L1 enhances response rates and that $\mathrm{PD}-\mathrm{L}_{1}{ }^{+}$macrophages suppress $\mathrm{T}$ cell proliferation under PD-1 and CTLA-4 blockade by a compensatory mechanism through CD80 binding.

As differences in PD-L1 expression were exclusively observed on intratumoral macrophages and were not present in the periphery, additional biomarkers will be required to monitor therapy response to ICB, especially in the context of glioblastoma patients. These biomarkers might address soluble factors of macrophage recruitment, polarization, and PD-L1-inducing factors in the blood. It moreover remains to be investigated if macrophagemediated resistance to ICB is acquired or preexisting, as a preexisting mechanism might be exploited to stratify and select patients that benefit from checkpoint blockade therapy using tumor samples.

In conclusion, this evidence suggests an important role of intratumoral macrophages-expressing PD-L1 and other immunosuppressing molecules in the response to PD-1 and CTLA-4 blockade, thereby inhibiting the induction of proliferation and reactivation of tumor-reactive $\mathrm{T}$ cells. Strategies to enhance therapy response to ICB might thus involve the mechanismdriven combination of ICB and targeting of TAMs.

\footnotetext{
Methods

Mice. C57Bl/6 J wild-type mice were purchased from Charles River or Janvier Laboratories at the age of 6-8 weeks. Animal procedures were performed in the accordance with all relevant ethical regulations for animal testing and research, and were approved by the governmental authorities (Regional Administrative Authority Karlsruhe, Germany). Sex- and age-matched mice were used for further experiments. If not stated otherwise, female mice were used for the experiments.
}

All mice were 7-12 weeks of age at use. Mice were kept under specific-pathogenfree (SPF) conditions at the animal facility of the DKFZ Heidelberg.

Cell culture. Gl261 cells were purchased from the National Cancer Institute. Gl261 cells were cultured in Dulbecco's modified Eagle's medium (DMEM) supplemented with $10 \%$ fetal bovine serum (FBS), $100 \mathrm{U} \mathrm{ml}^{-1}$ penicillin, and $100 \mu \mathrm{g}$ $\mathrm{ml}^{-1}$ streptomycin (Sigma-Aldrich) at $37^{\circ} \mathrm{C}, 5 \% \mathrm{CO}_{2}$. Gl261 cells were routinely tested for viral, mycoplasma, and non-murine cell contamination by multiplex cell contamination test (Multiplexion $\mathrm{GmbH})^{41}$. Primary murine $\mathrm{T}$ cells and myeloid cells were cultured in RPMI-1640 (Sigma-Aldrich) with $10 \%$ FBS, $100 \mathrm{U} \mathrm{ml}^{-1}$ penicillin, $100 \mu \mathrm{g} \mathrm{ml}^{-1}$ streptomycin, $25 \mathrm{mM}$ Hepes $\mathrm{pH} 7.4,1 \mathrm{mM}$ sodium pyr uvate, $5 \times 10^{-5} \mathrm{M} 2$-mercaptoethanol (Sigma-Aldrich), and $2 \mathrm{mM}$ L-glutamine (Thermo Fisher) at $37^{\circ} \mathrm{C}, 5 \% \mathrm{CO}_{2}$.

Gl261 tumor cell inoculation and tumor rechallenge. A total of $1 \times 10^{5} \mathrm{Gl} 261$ tumor cells were diluted in $2 \mu$ l sterile phosphate-buffered saline (PBS; SigmaAldrich) and stereotactically implanted into the right hemisphere of 7-9-week-old female $\mathrm{C} 57 \mathrm{Bl} / 6 \mathrm{~J}$ mice (coordinates: $2 \mathrm{~mm}$ right lateral of the bregma and $1 \mathrm{~mm}$ anterior to the coronal suture with an injection depth of $3 \mathrm{~mm}$ below the dural surface), using a $10 \mu \mathrm{l}$ Hamilton micro-syringe driven by a fine step stereotactic device (Stoelting). Tumor cell inoculation was performed under anesthesia and mice received analgesics for 2 days post operation. Mice were checked daily for tumor-related symptoms and sacrificed when tumor burden and stop criteria were met or mice showed signs of neurological deficit. For tumor rechallenge experiments 7-9-week-old male C57Bl/6 J mice (Charles River) were intracranially injected with $100.000 \mathrm{Gl} 261$ cells and mice were treated with anti-PD-1 + antiCTLA- 4 on day 14,17 , and 20 post inoculation. Tumor growth was monitored by MRI on day $14,21,29,42,70$, and 78 post inoculation. Responding mice were rechallenged with $1 \times 10^{5}$ Gl261 cells by intracranial injection into the contralateral hemisphere on day 57 post inoculation, as described above. In addition, $1 \times 10^{5}$ Gl261 cells were injected into a control group of five naive, age and sex-matched $\mathrm{C} 57 \mathrm{Bl} / 6 \mathrm{~J}$ mice. Mice were checked daily for tumor-related symptoms and sacrificed when tumor burden and stop criteria were met or mice showed signs of neurological deficit. Mice were followed for 63 days post tumor rechallenge (120 days after the first tumor injection) and survival was analyzed by Kaplan-Meier survival curves using log-rank Mantel-Cox test.

In vivo antibodies. For immune checkpoint therapy, $100 \mu \mathrm{g}$ anti-CTLA-4 (9D9, BioXCell) per mouse and $250 \mu$ anti-PD-1 (RMP1-14, BioXCell) per mouse or equivalent doses of isotype control antibodies (MCP-11 and 2A3, BioXCell) were administered by intraperitoneal (i.p.) injection in $200 \mu \mathrm{l}$ PBS on day 13, 16, and 19 after tumor inoculation. PD-L1 blockade $(200 \mu \mathrm{g}$ per mouse 10 F.9G2 or LTF-2 isotype control, BioXCell) was performed by i.p. injection in combination with anti-PD-1 and anti-CTLA-4 therapy on day 13,16, and 19 after tumor inoculation For PD-1 monotherapy, C57Bl/6 J mice were treated with $250 \mu \mathrm{g}$ anti-PD-1 or isotype control (C) on day 10,13 , and 16 and tumor growth was monitored by MR imaging on day 10,17 , and 24 post intracranial Gl261 tumor injection. For CD4 T cell blockade, $1000 \mu \mathrm{g}$ GK1.5 or LTF-2 isotype antibody per mouse were administered by i.p. injection on day 13 and 20 after tumor injection. CD8 T cell blockade was performed using $500 \mu \mathrm{g}$ anti-CD8 2.43 or LTF-2 isotype antibody per mouse on day $13,17,20$, and 24 after tumor inoculation. In CD4 and CD8 blocking experiments, ICB with anti-PD-1 and anti-CTLA- 4 was performed on day 15, 18, and 21 post inoculation to allow for $T$ cell depletion before therapy start. Efficacy of CD4 and CD8 depletion was confirmed before and during immune checkpoint therapy (every third day) by flow cytometry analysis of peripheral blood lymphocytes and by terminal flow cytometry analysis of TILs. For CSF1R blockade, $250 \mu \mathrm{g}$ AFS98 or $2 \mathrm{~A} 3$ isotype antibody per mouse were administered by i.p. injection on day $11,14,17,20,23$, and 25 after tumor injection. ICB with anti-PD1 and anti-CTLA-4 in combination with CSF1R depletion was performed on day 13,16 , and 19 post inoculation.

Survival experiments. For survival experiments, $1 \times 10^{5}$ Gl261 tumor cells were implanted into the right hemisphere of 7-9-week-old female $\mathrm{C} 57 \mathrm{Bl} / 6 \mathrm{~J}$ mice as described above. Mice were treated with anti-PD-1 + anti-CTLA-4 or isotype control on day 13,16, and 19 post inoculation and MRI was performed on day 13, 19 , and 26 as described above. Mice were checked daily for tumor-related symptoms and sacrificed when tumor burden and stop criteria were met or mice showed signs of neurological deficit. For survival data, data of two independent experiments were combined.

Tumor imaging and response criteria. MRI of Gl261 tumors was performed on day 13, 19, and 26 post tumor inoculation on a 9.4 Tesla horizontal bore small animal NMR scanner (BioSpec 94/20 USR, Bruker BioSpin GmbH) with a fourchannel phased-array surface receiver coil. MRI was performed under inhalation anesthesia with isoflurane. On day 13 post inoculation, mice were grouped according to tumor size. Tumor volumes and diameters were retrieved from standard T2-weighted sequences (TE: $33 \mathrm{~ms}$; TR: $2500 \mathrm{~ms}$ ) and tumor volume was manually segmented in the Osirix or ITKsnap imaging software in a blinded fashion regarding treatment condition. Treatment response was assessed analogous 
to the clinically established Immunotherapy Response Assessment in NeuroOncology (iRANO) criteria $^{15}$. Specifically, CR was defined as relative increase in lesion volume MRI1-MRI3 (\% $V_{\text {MRI3-MRII }}$ ) of $-100 \%$, PR as $\% V_{\text {MRI3-MRI1 }} \leq$ $-65.0 \%$ and $/$ or $\% V_{\text {MRI3-MRI }} \leq-65.0 \%$, SD as $\% V_{\text {MRI3-MRII }}>-65 \%$ and $<+40 \%$, and $\mathrm{PD}$ as $\% V_{\text {MRI3-MRI1 }} \geq+40 \%$. Mice with unconfirmed progression between MRI2 and MRI3 were defined as SD, if tumors regressed at least $30 \%$ between MRI2 and MRI3 (\% $V_{\text {MRI3-MRI2 }} \leq-30.0 \%$ ). Criteria for PD (NR) were met if tumor volume increased by $>=40 \%$ between MRI1 and MRI3 (thereby corresponding the $25 \%$ increase in the biperpendicular diameter mandated by the iRANO criteria, assuming spherical configuration of the tumor). Mice with $\mathrm{CR}, \mathrm{PR}$, or SD were defined as R mice. For validation of T2-weighted MR-based response evaluation, T2-w imaging data of ICB- and control-treated mice was compared to T1-w monitoring (T1-w parameters: after iv administration of $0.01 \mathrm{mmol}$ Gadoteric acid: RARE, coronal aquisition, matrix size $200 \times 200$, TE $6 \mathrm{~ms}$, TR $1000 \mathrm{~ms}$, two averages, flip angle $90^{\circ}$, refocusing angle $180^{\circ}$, resolution; $100 \mu \mathrm{m} \times 100 \mu \mathrm{m}$, slice thickness $0.7 \mathrm{~mm}$ ).

Radiomic signature discovery and response prediction. Radiomic analysis of MRI data was performed with an established workflow as described previously ${ }^{42,43}$. Briefly, radiomic features were calculated from the T2-hyperintense tumor volume from the first MRI and the change in features between the first and second MRI for radiomic signature discovery (Supplementary Data 1). Based on these radiomic features ( $n=423$ from each time point) gradient boosting machine-learning models were constructed to predict treatment failure at the third MRI. Model performance was evaluated using fivefold cross validation. In more detail, lesion volumes (MRI1 baseline lesion volumes, MRI2 during treatment lesion volumes, and MRI3 post treatment lesion volumes) were segmented on T2-weighted MR imaging using a region-growing segmentation algorithm implemented in ITKSNAP (www.itksnap.org). Radiomic features were calculated from these tumor segmentation masks from T2-weighted MR imaging for each mouse from both time points using the medical imaging interaction toolkit (MITK, www.mitk.org) ${ }^{44}$ This included (i) 146 first-order features (ii) 33 volume and shape features, (iii) 200 texture features, and 44 curvature features (CF). Next, a radiomic feature set consisting of all features from MRI1 as well as the absolute difference in each radiomic feature between time points MRI1 and MRI2 was used as an input for predictive modeling of treatment failure (i.e., prediction of response yes vs. no) at MRI3 (implemented using R version 3.5.1 (R Foundation for Statistical Computing, Vienna, Austria) with the caret library ${ }^{45}$ ). All radiomic features were $z$-score normalized (i.e., transformed to a mean of 0 and a standard deviation equal to 1 ). Predictive modeling was performed using a gradient boosting machine-learning algorithm that iteratively constructs an ensemble of weak decision tree learners through boosting to form a single strong predictive model (the tuning parameters (boosting iterations, max tree depth, shrinkage, and min. terminal node size) were automatically optimized via resampling procedures). The performance of the gradient boosting classifier was assessed based on a two-times repeated fivefold cross validation resampling procedure. The held-out predictions in each of the resampling iterations were used to calculate the accuracy, area under the receiver operating charasteristic (ROC), sensitivity, specificity, no information rate (largest class percentage for each molecular parameter, i.e., the prediction or accuracy by chance), and a hypothesis test (using the binom.test function) to evaluate whether the accuracy rate is greater than the no information rate. $P<0.05$ were considered significant.

Mutanome analysis GI261 tumors. DNA from Gl261 tumor tissue from R and NR mice was extracted using the INVISORB ${ }^{\oplus}$ DNA Tissue Mini Kit (STRATEC Biomedical AG) according to the manufacturer's instruction. RNA contamination was eliminated by RNase digestion with $10 \mathrm{mg} \mathrm{ml}^{-1}$ RNase at room temperature (RT) for $5 \mathrm{~min}$ (Sigma-Aldrich). Exome sequencing was performed on the Illumina NextSeq500 platform (Illumina Inc, San Diego, Calif.) using High output flow cell (75 nt reads paired end $+8 \mathrm{nt}$ index). SureSelectXT Target Enrichment System (Agilent Technologies) was used for library generation according to the manufacturer's instructions. To convert the vendor-specific sequencing data format generated by the Illumina NextSeq500 to a standard file format, the Illumina tool bcl2fastq (v2.15.0.4) ${ }^{46}$ was used. To check the sequencing read quality, reports were generated with the tool fastqc $(\mathrm{v} 0.10 .1)^{47}$. After quality checks the alignment was performed with bwa mem $(\mathrm{v} 0.7 .5)^{48}$ and the mouse reference genome GRCm 38.68 . The picard-tools (v1.105) ${ }^{49}$ were used to remove duplicates from the alignment files. The sorting and indexing of these files was done with samtools $(\mathrm{v} 0.1 .19)^{50}$. Afterward the variants were called by samtools mpileup (v0.1.19) for singlenucleotide variants and platypus (v0.7.9.1 $)^{51}$ for insertions and deletions. The basic annotations of the called variants was done with annovar (v2013-08-23) ${ }^{52}$

B16 tumor experiments. B16 melanoma cells were kindly provided by Günther J. Hämmerling (Division of Molecular Immunology, DKFZ Heidelberg). B16 cells were cultured in DMEM supplemented with $10 \% \mathrm{FBS}, 100 \mathrm{U} \mathrm{ml}^{-1}$ penicillin, and $100 \mu \mathrm{g} \mathrm{ml}^{-1}$ streptomycin (Sigma-Aldrich) at $37^{\circ} \mathrm{C}, 5 \% \mathrm{CO}_{2}$. B16 cells were routinely tested for viral, mycoplasma, and non-murine cell contamination by multiplex cell contamination test (Multiplexion $\mathrm{GmbH})^{41}$. For B16 tumor cell inoculation, cell suspension in PBS was mixed with an equal volume of Matrigel ${ }^{\circledR}$
Basement Membrane Matrix (Corning ${ }^{\circledR}$ ) and $5 \times 10^{4}$ cells in $200 \mu$ lof cell-matrix suspension were injected subcutaneously into the right flank of C57BL/6 J mice. Tumor growth was monitored by two-dimensional measurements using a caliper (area: width $\times$ length). A total of $100 \mu \mathrm{g}$ per mouse anti-CTLA-4 (9D9, BioXCell) and $250 \mu \mathrm{g}$ per mouse anti-PD-1 (RMP1-14, BioXCell) or equivalent doses of isotype control antibodies (MCP-11 and 2A3, BioXCell) were i.p. injected in $200 \mu \mathrm{l}$ PBS on day 7, 10, and 13 after tumor inoculation. Flow cytometry analysis of tumor-infiltrating and peripheral immune cells was performed on day 15 post inoculation.

CD3 Immunohistochemistry. Mice were sacrificed by cardial perfusion with PBS, excised brains were embedded in Tissue-Tek ${ }^{\circledR}$ O.C.T.TM (Sakura), and snapfrozen in cold 2-methylbutane (Sigma-Aldrich) on dry ice. Fresh-frozen sections were stained for $\mathrm{CD} 3$ with 1:100 rabbit anti-human/mouse CD3 (Dako; A 0452). In brief, cryo-sections were fixed with $4.5 \%$ paraformaldehyde and quenching of endogenous peroxidase was performed with $0.3 \% \mathrm{H}_{2} \mathrm{O}_{2}$. Sections were further washed and blocked with $4 \%$ normal goat serum in PBS at RT for $1 \mathrm{~h}$. CD3 was stained at $4{ }^{\circ} \mathrm{C}$ over night and secondary antibody incubation was performed with biotinylated goat anti-rabbit IgG (1:200; Vector; BA-1000) in $4 \%$ normal goat serum for $45 \mathrm{~min}$ at RT. After washing with PBS, the VECTASTAIN Elite ABC HRP Kit (Vector) was applied for $30 \mathrm{~min}$ at RT. Slides were washed with PBS and developed with 3,3'-Diaminobenzidine (DAB; Dako;). Reaction was stopped with $\mathrm{dH}_{2} \mathrm{O}$. Cryo-sections were further counterstained with hematoxylin for $3 \mathrm{~min}$ at RT and developed in tap water for $10 \mathrm{~min}$. Tissue sections were washed with $\mathrm{dH}_{2} \mathrm{O}$ followed by dehydration with 70\% EtOH, $96 \% \mathrm{EtOH}$, and $100 \% \mathrm{EtOH}$. Slides were cleared thrice with Histo-Clear at RT for 3 min and mounted with histomount medium. Images were acquired on Zeiss Cell Observer using the ZEN software. Quantitative analysis of $\mathrm{CD}^{+} \mathrm{T}$ cell numbers per $\mathrm{mm}^{2}$ tumor area was performed with ImageJ.

TCR sequencing and GLIPH analysis. DNA from TIL samples was extracted by QIAamp DNA Micro Kit (Qiagen; 56304) according to the manufacturer's instruction. TCR $\beta$ sequencing was performed using the TCR $\beta$ CDR3 Adaptive Biotechnologies ${ }^{\circledast}$ sequencing technology (immunoSEQTM Kit; Adaptive Biotechnologies; Seattle; WA $)^{53,54}$. Samples were sequenced on the Illumina NextSeq500 platform (Illumina Inc, San Diego, Calif.) using MID output flow cell $(156 \mathrm{nt}$ reads $+15 \mathrm{nt}$ Index). Data were analyzed with the ImmunoSEQ analyzer toolset and presented as productive amino acid sequences. Clonality was assessed by the percentage of the top ten frequent clones of all identified productive sequences, or productive clonality. Sequence similarity analysis was performed using R GLIPH analysis as adapted from Glanville et al. ${ }^{55}$. TCR sequences from healthy spleen and thymus from C57BL/6 J mice were used as reference database.

Processing of spleen, blood and tumor tissue. Spleens were excised and meshed twice through a $70 \mu \mathrm{m}$ cell strainer to obtain a single-cell suspension and erythrocytes were lysed with ACK buffer containing $150 \mathrm{mM} \mathrm{NH}_{4} \mathrm{Cl}, 10 \mathrm{mM} \mathrm{KHCO}_{3}$, and $100 \mu \mathrm{M} \mathrm{Na}_{2}$ EDTA. Blood samples were obtained by submandibular vein (immune cell monitoring during experiments) or cardial puncture in deep anesthesia (terminal immune cell analysis) and collected in syringes or tubes coated with $0.5 \mathrm{M}$ EDTA. Erythrocytes were lysed with ACK buffer and cells were washed twice and further processed for flow cytometry analysis. For isolation of TILs, mice were cardially perfused in deep anesthesia. For Gl261 tumors, the right hemisphere was excised and the cerebellum removed. For B16 tumors, flank tumors were excised. B16 tumors and Gl261-bearing hemispheres were mechanically dissected and enzymatically digested in HBSS (Sigma-Aldrich, 11088866001) supplemented with $50 \mu \mathrm{g} \mathrm{ml}^{-1}$ Liberase DL (Roche) under slow rotation at $37^{\circ} \mathrm{C}$ for $30 \mathrm{~min}$. Cells were subsequently meshed through a $100 \mu \mathrm{m}$ and $70 \mu \mathrm{m}$ cell strainer, stained, and analyzed by flow cytometry. For Gl261 cell suspensions, cells were purified using myelin removal beads II (Miltenyi Biotec; 130-096) according to the manufacturer's instruction.

Flow cytometry. For intracellular cytokine staining, cells were incubated with $5 \mu \mathrm{g}$ $\mathrm{ml}^{-1}$ Brefeldin A (Sigma-Aldrich) for $5 \mathrm{~h}$ at $37^{\circ} \mathrm{C}, 5 \% \mathrm{CO}_{2}$ to allow for intracellular enrichment of cytokines. Brain tumor and spleen cell suspensions were blocked with anti-CD16/CD32 (eBioscience; 93; 14-0161) and extracellular targets were stained at $4^{\circ} \mathrm{C}$ for $30 \mathrm{~min}$ (Supplementary Data 2). Intracellular antigens were fixed, permeabilized, and stained using the FOXP3/transcription factor staining buffer set (eBioscience; 00-5523) and the antibodies listed in Supplementary Data 2. Staining of intracellular targets was performed for $45 \mathrm{~min}$ at $4{ }^{\circ} \mathrm{C}$. Stained lymphocytes were analyzed on FACS Canto II (BD Biosciences; Germany) or on Attune NxT (Thermo Fisher; Germany). FlowJo V9 or V10 were used for data analysis. Multiparameter FACS data were generated on a FACSSymphony (BD Biosciences) using the antibodies described in Supplementary Data 2. Data were compensated, exported (FlowJo V10), uploaded, and normalized using Cyt3 (Matlab_R2018b). The new generated FCS files were uploaded in Rstudio (Version 1.1.463). tSNE (displaying stochastically selected events from all different conditions) and FlowSOM (events from each condition) were performed as described by Brumelman et al. ${ }^{56}$. 
Ex vivo phagocytosis. For isolation of $\mathrm{CD} 11 \mathrm{~b}^{+}$cells of Gl261 tumors from ICB R, ICB NR, and C mice, myelin was removed of tumor single-cell suspension with myelin removal beads II (Miltenyi Biotec; 130-096) according to the manufacturer's instruction. Subsequently, CD11 $\mathrm{b}^{+}$cells were purified using MagniSort ${ }^{\mathrm{tm}}$ Mouse CD11b Positive Selection Kit (eBioscience; 8802-6860-74). Ex vivo phagocytosis of $\mathrm{CD}_{11 \mathrm{~b}^{+}}$cells was assessed as previously described ${ }^{22}$. In brief, $\mathrm{CD} 11 \mathrm{~b}^{+}$ cells were plated onto ultra-low attachment 96-well plates (Corning) and incubated at $37^{\circ} \mathrm{C}, 5 \% \mathrm{CO}_{2}$ for $20 \mathrm{~min}$ to allow for cell resting. $\mathrm{CD} 11 \mathrm{~b}^{+}$cells were subsequently cultured at $37^{\circ} \mathrm{C}, 5 \% \mathrm{CO}_{2}$ for $2 \mathrm{~h}$ with pHrodo ${ }^{\text {mox }}$-red Staphylococcus aureus BioParticles (Thermo Fisher) according to the manufacturer's instruction. Phagocytosis was assessed by flow cytometry analysis for pHrodo-red ${ }^{+}$cells of macrophages $\left(\mathrm{CD} 45^{\text {high }} \mathrm{CD} 1 \mathrm{~b}^{+}\right.$cells) and microglia $\left(\mathrm{CD} 45^{\text {low }} \mathrm{CD} 11 \mathrm{~b}^{+}\right.$cells). PD-L1 was blocked during incubation with pHrodo ${ }^{\text {mo }}$-red S. aureus BioParticles with $20 \mu \mathrm{g}$ $\mathrm{ml}^{-1}$ anti-PD-L1 (10 F.9G2; BioXCell).

Apoptosis of intratumoral macrophages. Macrophages $\left(\mathrm{CD} 45^{\text {high }} \mathrm{CD} 11 \mathrm{~b}^{+}\right)$of ICB R and ICB NR were stained with annexin V-FITC (BioVision, 1:100) and DAPI (1:250, Invitrogen, Carlsbad, USA) in Annexin V binding buffer

(eBioscience, Germany) at RT for $15 \mathrm{~min}$, and analyzed using BD-FACS Canto II. Early apoptosis was defined by single annexin $\mathrm{V}$ positivity. Late apoptosis was defined as annexin $\mathrm{V}$ and DAPI double positivity.

Blood immune cell monitoring. Blood samples of ICB-treated mice were collected on day 15 and 21 after Gl261 inoculation using submandibular vein puncture and collected in tubes coated with $0.5 \mathrm{M}$ EDTA. Erythrocytes were lysed with ACK buffer containing $150 \mathrm{mM} \mathrm{NH}_{4} \mathrm{Cl}, 10 \mathrm{mM} \mathrm{KHCO}$, and $100 \mu \mathrm{M} \mathrm{Na}_{2}$ EDTA. Cells were washed twice with PBS and further processed for flow cytometry analysis.

Plasma cytokine array. Blood samples were collected by submandibular vein puncture and plasma was obtained by centrifugation at $2000 \times g$, RT for $10 \mathrm{~min}$. Plasma cytokine analysis was performed with pooled plasma samples at equal ratios for five mice per group according to the manufacturer's instructions (Proteome Profiler ${ }^{\text {rnt }}$ Array Mouse Cytokine Array Panel A; R\&D Systems; ARY006). Samples were measured on the ChemiDocTM MP Blot reader system (BioRad; Hercules, Calif.). ImageJ 1.48 and the Gilles Carpentier's Protein Array Analyzer for ImageJ toolset were used for data analysis.

Ex vivo and in vitro $\mathbf{T}$ cell suppression. For ex vivo and in vitro $\mathrm{T}$ cell suppression assays, $\mathrm{T}$ cells were purified from spleens of naive C57BL/6 J mice using the MagniSort $^{\text {tw }}$ Mouse T cell Enrichment Kit (eBioscience; 8802-6820), labeled with $5 \mu \mathrm{M}$ carboxyfluorescein succinimidyl ester (CFSE; Thermo Fisher; C34570) and preactivated prior to myeloid cell co-culture with plate-bound $0.1 \mu \mathrm{g} \mathrm{ml}^{-1}$ anti-CD3 (145-2C11; eBioscience;) and $1 \mu \mathrm{g} \mathrm{ml}^{-1}$ anti-CD28 (37.51; Biolegend) at $37^{\circ} \mathrm{C}, 5 \%$ $\mathrm{CO}_{2}$ for 16-18 h. Ex vivo $\mathrm{T}$ cell suppression assay with tumor-associated myeloid cells was adapted from De Henau et al. ${ }^{18}$. In brief, Gl261-associated myeloid cells were isolated of from ICB R, ICB NR, and C mice. To this end, single-cell suspensions of tumor-bearing hemispheres were subjected to myelin removal (Myelin removal beads II; Miltenyi Biotec; 130-096) and CD11 b cells were purified by MACS using the MagniSort ${ }^{\text {tm }}$ Mouse CD11b Positive Selection Kit (eBioscience;

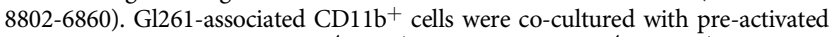
$\mathrm{T}$ cells at a ratio of $1: 1\left(2.5 \times 10^{4} \mathrm{CD}^{+} \mathrm{T}\right.$ cells and $2.5 \times 10^{4} \mathrm{CD}^{1} 1 \mathrm{~b}^{+}$myeloid cells $)$ in murine T cell proliferation medium at $37^{\circ} \mathrm{C}, 5 \% \mathrm{CO}_{2}$ for $72 \mathrm{~h}$. T cell proliferation was examined by CFSE mean fluorescence intensity of living $\mathrm{CD} 3^{+} \mathrm{CD} 8^{+}$and living $\mathrm{CD}^{+}{ }^{+} \mathrm{CD} 4^{+} \mathrm{T}$ cells, and percentage of cells per cell division

Ex vivo TIL cytotoxicity. For ex vivo cytotoxicity analysis of ICB R, ICB NR, and C Gl261 TILs, a lactate dehydrogenase (LDH) release assay was applied (Promega; G1780). A total of $5 \times 10^{3} \mathrm{Gl} 261$ cells were seeded onto 96 -well flat bottom plates and incubated at $37^{\circ} \mathrm{C}, 5 \% \mathrm{CO}_{2}$ over night to allow for tumor cell adherence. For isolation and purification of TILs from ICB R, ICB NR, and C-treated Gl261 tumors on day 27 post inoculation, tumor-bearing hemispheres were processed to single-cell suspensions, and TILs were purified with myelin removal beads II (Miltenyi Biotec; 130-096) and MagniSort ${ }^{\text {tw }}$ Mouse CD3 Positive Selection Kit (eBioscience; 8802-6840) according to the manufacturer's instruction. Purified TILs were co-cultured with Gl261 cells at a ratio of $10: 1\left(5 \times 10^{4} \mathrm{CD}^{+} \mathrm{T}-5 \times 10^{3}\right.$ Gl261 cells) at $37^{\circ} \mathrm{C}, 5 \% \mathrm{CO}_{2}$ for $4 \mathrm{~h}$. A total of $4-5$ ICB R, ICB NR, and C TIL samples per group were pooled for cytotoxicity analysis. LDH release of TILmediated Gl261 killing was assessed with the CytoTox $96^{\circledR}$ Non-Radioactive Cytotoxicity Kit (Promega) according to the manufacturer's instruction and OD was measured on a iMarkTM Microplate reader (BioRad; Hercules, Calif.) at $490 \mathrm{~nm}$. Values were corrected for spontaneous effector and target cell LDH release and cell culture medium background. Data are represented as tumor cell lysis relative to positive Gl261 lysis control.

NanoString analysis and inflammatory gene signatures. RNA from FACS sorted macrophages $\left(\mathrm{CD} 45^{\text {high }} \mathrm{CD} 1 \mathrm{~b}^{+}\right.$) of Gl261 tumors of ICB R and ICB NR mice was extracted using the PicoPureTM RNA Isolation Kit (Arcturus; KIT0202) and gene expression analysis was performed using the nCounter Mouse
Immunology Panel (NanoString; XT-CSO-MIM1-12) with the nCounter NanoString $^{\text {tw }}$ technology (NanoString Technologies; Seattle, WA ${ }^{57}$. RNA input per sample was $25 \mathrm{ng}$. Data analysis were performed by nSolver 3.0. Marker for proand anti-inflammatory gene signatures were selected according to previously described marker. Gene signature scores were calculated as geometric mean of each gene expression. Controls and low count genes were removed from the NanoString count matrix, followed by a scalar normalization and variance modeling 58,59 . Differential gene expression analysis was performed by an eBayes adjusted moderated $t$-statistic linear regression model ${ }^{60}$. Pro- and anti-inflammatory metagene signatures were generated from previously reported markers (Supplementary Table 1) and the geometric mean was estimated in each sample for the different signatures.

CIBERSORT analysis human glioblastoma. CIBERSORT analysis was applied to expression data of pre-ICB (pembrolizumab/nivolumab) GBM tissue from Zhao et $\mathrm{al}^{25}$. Expression data from GBM samples that were obtained more than 6.5 months prior to the first ICB therapy (pembrolizumab/nivolumab) were excluded. Response classification of patients was adapted from Zhao et al. ${ }^{25}$. In detail, response criteria were met when samples after PD-1 inhibitor therapy showed signs of pseudoprogression (inflammatory response with very few or no tumor cells detectable) or stable or continually shrinking tumor lesions over a minimum of 6 months as detected by $\mathrm{MRI}^{25}$. RNAseq *.fastq files for selected patients were downloaded from the ENA using the Aspera Connect client. Reads were aligned to the human genome (GRCh38) using STAR (2.7.0c), and a gene expression matrix (as TPM) was generated using RSEM. The gene expression matrix was analyzed by CIBERSORT using the LM22 signature gene file of 22 immune cell types ${ }^{61}$. Immune cell subtype proportions were compared using Wilcoxon rank-sum test (WRST) and false discovery rate adjustment was performed by Benjamini and Hochberg correction.

Immunogram ICB response. Fold changes (R/NR) of response features were $\log 2$ transformed and $z$ transformation for all features was applied. Data are presented as a radar chart.

Statistics. Data are represented as individual values or as mean \pm SEM. Group sizes $(n)$ and applied statistical tests are indicated in figure legends. Significance was assessed by either unpaired $t$-test analysis, paired $t$-test analysis,or one-way analysis of variance (ANOVA) analysis with Tukey, Dunnett or Sidak post hoc testing as indicated in figure legends. Spearman correlation was applied for all correlation analysis and the Kaplan-Meier method was used to examine survival differences. Statistics were calculated using GraphPad Prism 7.0.

Reporting summary. Further information on research design is available in the Nature Research Reporting Summary linked to this article.

\section{Data availability}

RNA-seq data that support the findings of this study has been deposited in the GEO repository (GSE129877) and will be made available prior to publication. All additional data sets generated or analyzed during this study are included in this published article and supplementary information files. Data underlying CIBERSORT analysis was published by Zhao J et al. ${ }^{25}$. (Nat. Med., 2019) and was accessed via the GEO repository (GSE121810). The source data underlying Figs. $1 b-j, 2 a-f, 3 a-h, 4 a-c, 4 e, f, 5 a-f$, and Supplementary Figs. 1a-g, 2a-c, 3a, 3c, d, 4a, 4c-f, 6a-g, 7a, b, 8a-c, 9a-c, 10a-d, and $11 \mathrm{~b}-\mathrm{f}$ are provided as a Source Data file.

\section{Code availability}

The full codes of all scripts are available on reasonable request. For further information on software package versions please refer to the Nature Research Reporting Summary linked to this article.

Received: 25 November 2019; Accepted: 16 January 2020; Published online: 18 February 2020

\section{References}

1. Pardoll, D. M. The blockade of immune checkpoints in cancer immunotherapy. Nat. Rev. Cancer 12, 252-264 (2012).

2. Larkin, J. et al. Combined nivolumab and ipilimumab or monotherapy in untreated melanoma. N. Engl. J. Med. 373, 23-34 (2015).

3. Gettinger, S. et al. Nivolumab monotherapy for first-line treatment of advanced non-small-cell lung cancer. J. Clin. Oncol. 34, 2980-2987 (2016)

4. Goldberg, S. B. et al. Pembrolizumab for patients with melanoma or nonsmall-cell lung cancer and untreated brain metastases: early analysis of a nonrandomised, open-label, phase 2 trial. Lancet Oncol. 17, 976-983 (2016). 
5. Tawbi, H. A. et al. Combined nivolumab and ipilimumab in melanoma metastatic to the brain. N. Engl. J. Med. 379, 722-730 (2018).

6. Reardon, D. A. et al. OS10.3 randomized phase 3 study evaluating the efficacy and safety of nivolumab vs bevacizumab in patients with recurrent glioblastoma: CheckMate 143. Neuro Oncol. 19, iii21-iii21 (2017).

7. Johanns, T. M. et al. Immunogenomics of Hypermutated Glioblastoma: a patient with germline POLE deficiency treated with checkpoint blockade immunotherapy. Cancer Discov. 6, 1230-1236 (2016).

8. Hodges, T. R. et al. Mutational burden, immune checkpoint expression, and mismatch repair in glioma: implications for immune checkpoint immunotherapy. Neuro Oncol. 19, 1047-1057 (2017).

9. Wang, Q. et al. Tumor evolution of glioma-intrinsic gene expression subtypes associates with immunological changes in the microenvironment. Cancer Cell 33, 152 (2018).

10. Thorsson, V. et al. The immune landscape of cancer. Immunity 48, 812-830. e14 (2018)

11. Roth, P., Valavanis, A. \& Weller, M. Long-term control and partial remission after initial pseudoprogression of glioblastoma by anti-PD-1 treatment with nivolumab. Neuro Oncol. 19, 454-456 (2017).

12. Bouffet, E. et al. Immune checkpoint inhibition for hypermutant glioblastoma multiforme resulting from germline biallelic mismatch repair deficiency. $J$. Clin. Oncol. 34, 2206-2211 (2016).

13. Johanns, T. M. et al. Endogenous neoantigen-specific CD8 T cells identified in two glioblastoma models using a cancer immunogenomics approach. Cancer Immunol. Res. 4, 1007-1015 (2016).

14. Genoud, V. et al. Responsiveness to anti-PD-1 and anti-CTLA-4 immune checkpoint blockade in SB28 and GL261 mouse glioma models. OncoImmunology 7, e1501137 (2018).

15. Okada, H. et al. Immunotherapy Response Assessment in Neuro-Oncology (iRANO): a report of the RANO working group. Lancet Oncol. 16, e534-e542 (2015).

16. Reardon, D. A. et al. Glioblastoma eradication following immune checkpoint blockade in an orthotopic, immunocompetent model. Cancer Immunol. Res. 4, 124-135 (2016).

17. Arlauckas, S. P. et al. In vivo imaging reveals a tumor-associated macrophagemediated resistance pathway in anti-PD-1 therapy. Sci. Transl. Med. 9, eaal3604 (2017).

18. De Henau, O. et al. Overcoming resistance to checkpoint blockade therapy by targeting PI3K $\gamma$ in myeloid cells. Nature 539, 443-447 (2016).

19. Butowski, N. et al. Orally administered colony stimulating factor 1 receptor inhibitor PLX3397 in recurrent glioblastoma: an Ivy Foundation Early Phase Clinical Trials Consortium phase II study. Neuro Oncol. 18, 557-564 (2016).

20. Pyonteck, S. M. et al. CSF-1R inhibition alters macrophage polarization and blocks glioma progression. Nat. Med. 19, 1264-1272 (2013).

21. Wurdinger, T., Deumelandt, K., van der Vliet, H. J., Wesseling, P. \& de Gruijl, T. D. Mechanisms of intimate and long-distance cross-talk between glioma and myeloid cells: How to break a vicious cycle. Biochim. Biophys. Acta 1846, 560-575 (2014).

22. Gordon, S. R. et al. PD-1 expression by tumour-associated macrophages inhibits phagocytosis and tumour immunity. Nature 545, 495-499 (2017).

23. Shen, L. et al. PD-1/PD-L pathway inhibits M.tb-specific CD4(+) T-cell functions and phagocytosis of macrophages in active tuberculosis. Sci. Rep. 6, 38362 (2016)

24. Butte, M. J., Keir, M. E., Phamduy, T. B., Freeman, G. J. \& Sharpe, A. H. PDL1 interacts specifically with B7-1 to inhibit T cell proliferation. Immunity 27, 111-122 (2007).

25. Zhao, J. et al. Immune and genomic correlates of response to anti-PD-1 immunotherapy in glioblastoma. Nat. Med. 25, 462-469 (2019).

26. Cloughesy, T. F. et al. Neoadjuvant anti-PD-1 immunotherapy promotes a survival benefit with intratumoral and systemic immune responses in recurrent glioblastoma. Nat. Med. 25, 477-486 (2019).

27. Schalper, K. A. et al. Neoadjuvant nivolumab modifies the tumor immune microenvironment in resectable glioblastoma. Nat. Med. 25, 470-476 (2019).

28. Kickingereder, P. et al. Automated quantitative tumour response assessment of MRI in neuro-oncology with artificial neural networks: a multicentre, retrospective study. Lancet Oncol. https://doi.org/10.1016/S1470-2045(19) 30098-1 (2019).

29. Parsa, A. T. et al. Loss of tumor suppressor PTEN function increases B7-H1 expression and immunoresistance in glioma. Nat. Med. 13, 84-88 (2007).

30. Wintterle, S. et al. Expression of the B7-related molecule B7-H1 by glioma cells: a potential mechanism of immune paralysis. Cancer Res. 63, 7462-7467 (2003).

31. Ricklefs, F. L. et al. Immune evasion mediated by PD-L1 on glioblastomaderived extracellular vesicles. Sci. Adv. 4, eaar2766 (2018).

32. Berghoff, A. S. et al. Programmed death ligand 1 expression and tumorinfiltrating lymphocytes in glioblastoma. Neuro Oncol. 17, 1064-1075 (2015).

33. Garber, S. T. et al. Immune checkpoint blockade as a potential therapeutic target: surveying CNS malignancies. Neuro Oncol. 18, 1357-1366 (2016).
34. Nduom, E. K. et al. PD-L1 expression and prognostic impact in glioblastoma. Neuro Oncol. 18, 195-205 (2016).

35. Antonios, J. P. et al. Immunosuppressive tumor-infiltrating myeloid cells mediate adaptive immune resistance via a PD-1/PD-L1 mechanism in glioblastoma. Neuro Oncol. 19, 796-807 (2017).

36. Bloch, O. et al. Autologous heat shock protein peptide vaccination for newly diagnosed glioblastoma: impact of peripheral pd-l1 expression on response to therapy. Clin. Cancer Res. 23, 3575-3584 (2017).

37. Patel, S. J. et al. Identification of essential genes for cancer immunotherapy. Nature 548, 537-542 (2017).

38. Gabrusiewicz, K. et al. Glioblastoma stem cell-derived exosomes induce M2 macrophages and PD-L1 expression on human monocytes. Oncoimmunology 7, e1412909 (2018).

39. Lamano, J. B. et al. Glioblastoma-derived IL-6 induces immunosuppressive peripheral myeloid cell PD-L1 and promotes tumor growth. Clin. Cancer Res. 25, 3643--3657 (2019).

40. Lee, Y.-J. et al. Macrophage PD-L1 strikes back: PD-1/PD-L1 interaction drives macrophages toward regulatory subsets. Adv. Biosci. Biotechnol. 04, 19 (2013).

41. Schmitt, M. \& Pawlita, M. High-throughput detection and multiplex identification of cell contaminations. Nucleic Acids Res. 37, e119 (2009).

42. Kickingereder, P. et al. Large-scale radiomic profiling of recurrent glioblastoma identifies an imaging predictor for stratifying anti-angiogenic treatment response. Clin. Cancer Res. 22, 5765-5771 (2016).

43. Kickingereder, P. et al. Radiomic subtyping improves disease stratification beyond key molecular, clinical, and standard imaging characteristics in patients with glioblastoma. Neuro Oncol. 20, 848-857 (2018).

44. Nolden, M. et al. The Medical Imaging Interaction Toolkit: challenges and advances: 10 years of open-source development. Int. J. Comput. Assist. Radiol. Surg. 8, 607-620 (2013).

45. Kuhn, M. Building predictive models in $\mathrm{R}$ using the caret package. J. Stat. Softw. 28, 1-26 (2008).

46. Illumina, "bcl2fastq." https://support.illumina.com/sequencing/ sequencing_software/bcl2fastq-conversion-software.html (2019).

47. Andrew S. "FastQC: a quality control tool for high throughput sequence data." http://www.bioinformatics.babraham.ac.uk/projects/fastqc (2019).

48. Li, H. Aligning sequence reads, clone sequences and assembly contigs with BWA-MEM. https://arxiv.org/abs/1303.3997 (2013).

49. GitHub Repository. Broad Institute, "Picard Toolkit.” http://broadinstitute. github.io/picard/ (2018).

50. Li, H. et al. The Sequence Alignment/Map format and SAMtools. Bioinform Oxf. Engl. 25, 2078-2079 (2009)

51. GitHub Repository. Rimmer A. "Platypus." https://github.com/andyrimmer/ Platypus.

52. Wang, K., Li, M. \& Hakonarson, H. ANNOVAR: functional annotation of genetic variants from high-throughput sequencing data. Nucleic Acids Res. 38 e164 (2010).

53. Carlson, C. S. et al. Using synthetic templates to design an unbiased multiplex PCR assay. Nat. Commun. 4, 2680 (2013).

54. Pollack Seth, M. et al. T-cell infiltration and clonality correlate with programmed cell death protein 1 and programmed death-ligand 1 expression in patients with soft tissue sarcomas. Cancer 123, 3291-3304 (2017).

55. Glanville, J. et al. Identifying specificity groups in the $\mathrm{T}$ cell receptor repertoire. Nature 547, 94-98 (2017).

56. Brummelman, J. et al. Development, application and computational analysis of high-dimensional fluorescent antibody panels for single-cell flow cytometry. Nat. Protoc. 14, 1946-1969 (2019).

57. Wohlfarth, C. et al. miR-16 and miR-103 impact 5-HT 4 receptor signalling and correlate with symptom profile in irritable bowel syndrome. Sci. Rep. 7, 14680 (2017).

58. Robinson, M. D. \& Oshlack, A. A scaling normalization method for differential expression analysis of RNA-seq data. Genome Biol. 11, R25 (2010)

59. Law, C. W., Chen, Y., Shi, W. \& Smyth, G. K. voom: precision weights unlock linear model analysis tools for RNA-seq read counts. Genome Biol. 15, R29 (2014).

60. Ritchie, M. E. et al. limma powers differential expression analyses for RNAsequencing and microarray studies. Nucleic Acids Res. 43, e47 (2015).

61. Newman, A. M. et al. Robust enumeration of cell subsets from tissue expression profiles. Nat. Methods 12, 453 (2015).

\section{Acknowledgements}

We thank Katharina Rauschenbach, L.W., K.J., A.v.L., M.F., J.M., and Mirjam Lutz for expert technical assistance in MRI, tissue processing, and sequencing. We acknowledge the support by the Center for Preclinical Research, the Core Facility for Small Animal Imaging and Flow Cytometry at the German Cancer Research Center, and the Flow Cytometry Core Facility at the Medical Faculty Mannheim of the Heidelberg University. We thank the nCounter Core Facility Heidelberg for providing the nCounter system and related services. We acknowledge the support in biostatistics by Tim Holland-Letz. B16 
melanoma cells were kindly provided by Günther J. Hämmerling (Division of Molecular Immunology, DKFZ Heidelberg). This work was supported by grants from the DKFZMOST program (project number 2526) and the Helmholtz Gemeinschaft, Zukunftsthema "Immunology and Infection" (ZT0027), the Dr. Rolf M. Schwiete Foundation and the Sonderförderlinie 'Neuroinflammation' of the Ministry of Science of Baden Württemberg and the German Ministry of Education and Science (National Center for Tumor Diseases Heidelberg NCT 3.0 program 'Precision immunotherapy of brain tumors' and the DKTK program) and by grants from the Deutsche Forschungsgemeinschaft (DFG; project C01 within CRC1366 "Vascular Control of Organ Function" (project number 39404578) and project B01 within CRC1389 "Understanding and targeting resistance in glioblastoma” (project number 404521405) to M.P., the Helmholtz International Graduate School for Cancer Research to J.K.S, M.K, and K.S., the German-Israeli Helmholtz Research School in Cancer Biology to K.A. and fellowships from the Mildred-Scheel doctoral program of the German Cancer Aid to J.B. and V.T. M.O.B. was supported by the Else Kröner Fresenius Stiftung (2017-A25). This work was further supported by grants from the Swiss Cancer league (BB), Swiss National Science Foundation (PP00P3_144781 to M.G. and 310030_146130, 316030_150768, and 310030_170320 to B.B.), the European Union FP7 Project ATECT (to B.B.), Forschungskredit Postdoc University of Zurich (to D.D.F.), and the University Research Priority Project Translational Cancer Research (to B.B., A.R.L., and N.G.N.).

\section{Author Contributions}

K.A., V.T., J.B., and M.P. designed the experiments and analyzed data. K.A., V.T., J.B., J.K.S., Mi.F., K.S., M.K., F.C., L.W., K.J., A.v.L., and L.B. performed in vivo and ex vivo experiments. Ma.F., A.A., K.P., and M.O.B. performed MRI and data analysis. A.R.L., N.G.N., and D.D.F performed and processed multiparameter flow cytometer analysis. F.S., D.S., J.M., E.G., and A.S. processed and analyzed sequencing data. R.R. performed NanoString expression analysis. P.K. and G.B. established the radiomic signature and performed radiomic response prediction. B.N., A.v.D., C.O., S.H., M.B., W.W., and B.B. were involved in data interpretation. K.A. and M.P. wrote the manuscript with input from all co-authors.

\section{Competing interests}

The authors declare no competing interests.

\section{Additional information}

Supplementary information is available for this paper at https://doi.org/10.1038/s41467020-14642-0.

Correspondence and requests for materials should be addressed to M.P.

Peer review information Nature Communications thanks the anonymous reviewer(s) for their contribution to the peer review of this work.

Reprints and permission information is available at http://www.nature.com/reprints

Publisher's note Springer Nature remains neutral with regard to jurisdictional claims in published maps and institutional affiliations.

(c) (i) Open Access This article is licensed under a Creative Commons Attribution 4.0 International License, which permits use, sharing, adaptation, distribution and reproduction in any medium or format, as long as you give appropriate credit to the original author(s) and the source, provide a link to the Creative Commons license, and indicate if changes were made. The images or other third party material in this article are included in the article's Creative Commons license, unless indicated otherwise in a credit line to the material. If material is not included in the article's Creative Commons license and your intended use is not permitted by statutory regulation or exceeds the permitted use, you will need to obtain permission directly from the copyright holder. To view a copy of this license, visit http://creativecommons.org/ licenses/by/4.0/.

(C) The Author(s) 2020 